\title{
Design and Analysis of Transmission Strategies in Channel-Hopping Cognitive Radio Networks
}

\author{
Chi-Mao Lee, Jia-Shi Lin, Student Member, IEEE, \\ Kai-Ten Feng, Member, IEEE, and Chung-Ju Chang, Fellow, IEEE
}

\begin{abstract}
In recent years, channel-hopping-based medium access control protocols have been proposed to improve the capacity in a decentralized multichannel cognitive radio $(\mathrm{CR})$ network without using extra control channels. Each CR user has to stochastically follow a default channel-hopping sequence in order to locate a channel and conduct its frame transmission. In this paper, theoretical analysis is conducted on the probability of channel availability and the average frame delay for primary users (PUs) by considering the impact caused by imperfect sensing of CR users and imperfect synchronization between the primary and CR networks. According to the proposed analytical model with realistic considerations, an optimal channel-hopping sequence (OCS) approach is designed for the CR users based on a dynamic programming technique. It is designed by exploiting the optimal load balance between channel availability and channel utilization within the delay constraints of PUs. By adopting the OCS approach, maximum aggregate throughput of CR users can be achieved while considering PU's quality-of-service (QoS) requirements. Moreover, in addition to the paired CR networks, the logical partition problem that occurs in generalized CR networks will also be addressed. This problem can severely degrade the aggregate throughput due to the decreased probability of connectivity between CR users, especially in a CR network with heavy traffic. Therefore, both wake-up successive contention (WSC) and wake-up counter-reset successive contention (WCSC) algorithms are proposed to increase the number of negotiations by both exploring the blind spot of imperfect sensing and amending the contention mechanisms between CR users. Compared to conventional channel-hopping sequences, numerical results illustrate that the proposed approaches can effectively maximize aggregate throughput for CR users under the QoS requirements of PUs.
\end{abstract}

Index Terms-Cognitive radio, queuing networks, channel-hopping sequence, dynamic programming.

\section{INTRODUCTION}

$\mathrm{T}$ HE increasing demand for spectrum resources has caused the so-called spectrum scarcity problem primarily because of the conventional approaches of static spectrum allocation. According to FCC regulations [1], a large portion of the priced frequency spectrum is underutilized in most times and locations, which are known as spectrum holes. Consequently, cognitive radio (CR) for dynamic spectrum access (DSA) has been exploited for more efficient spectrum utilization over the licensed bands [2] such as the IEEE 802.22 [3], [4] standard. This is an emerging standard that allocates frequency spectrums for TV broadcast services via a license-exempt basis. The CR user (CRU), i.e., unlicensed user, is capable of sensing channel conditions and can adapt internal parameters to access licensed channels while these channels are not being utilized by primary users (PUs), i.e., licensed users. In addition to the IEEE 802.22 standard which focuses on specifications for a centralized CR network, there are also a great number of studies interested in decentralized DSA in multichannel TDMA-based, i.e., time slotted-based, primary networks. The main focus is on designing a medium access control

- The authors are with the Department of Electrical Engineering, National Chiao Tung University, 1001 Ta Hsueh Road, Hsinchu 30049, Taiwan, ROC. E-mail: frundly@gmail.com, uxoxox.cm96g@g2.nctu.edu.tw, $\{k t f e n g$, cjchang\}@mail.nctu.edu.tw.

Manuscript received 24 Aug. 2010; revised 26 July 2011; accepted 25 Aug. 2011; published online 27 Sept. 2011.

For information on obtaining reprints of this article, please send e-mail to: tmc@computer.org, and reference IEEECS Log Number TMC-2010-08-0397. Digital Object Identifier no. 10.1109/TMC.2011.205.
(MAC) protocol to effectively exploit channel availability under the overlay paradigm, considering that both PUs and CRUs cannot transmit data simultaneously [5].

These MAC protocols can be categorized into two different types of schemes according to their access strategies, including sensing-based and probability-based methods. In sensing-based schemes [6], [7], the CRUs have to sense part of the channels before deciding which channel to access. In general, this will lead to higher channel utilization and reduced complexity in the handshaking mechanism since the hidden multichannel problem [8] is alleviated. However, this method has been described as impractical [9], [10], [11] because each CRU must equip multiple transceivers to conduct spectrum sensing. It is also inefficient for a CRU to sense and switch among the entire frequency spectrums within a slot time in order to obtain the required spectrum map knowledge. On the other hand, with probability-based schemes, the CRUs have to decide which channel to sense according to certain statistical information from the PUs and subsequently transmit their data. This type of scheme is implemented to amend the problem arising with sensing-based protocols that each CRU is only required to possess a single transceiver for channel sensing. Therefore, it is important for the CRUs to accurately acquire the opportunities to access idle channels in an efficient manner.

In [12], an opportunistic spectrum access MAC protocol is proposed by utilizing a common control channel for the CRUs to both negotiate the channel reservation and to determine which channel to sense based on the stationary 
idle probability of the PU in each channel. However, the usage of an additional common control channel is still controversial. The partially observable Markov decision process (POMDP) framework in [9] provides an optimal sensing strategy for a CRU to select and sense a channel that has the highest probability of maximizing the network throughput. However, the proposed framework is computationally complex and is not suitable for networks with multiple CRUs. In [13], an optimal channel sensing policy for more practical CR networks is explored by considering imperfect global synchronization between the $C R$ and primary networks. The Munkres algorithm with low computational complexity is adopted to solve the optimization problem in channel selection. However, the proposed scheme is appropriate for CR networks with only a single pair of CRUs to communicate with one another. On the other hand, channel-hopping based protocols are proposed in [8], [11], [14] where multiple pairs of CRUs switch among the licensed channels with their distinct default channelhopping sequences. When a CR transmitter wants to communicate with its intended $C R$ receiver, the $C R$ transmitter changes its hopping schedule and follows the channel-hopping sequence of the intended receiver in order to conduct negotiation and transmit the data if the channel is not currently utilized by the PU. However, the channelhopping sequences in these schemes are uniformly generated in each channel, which is appropriate only for homogeneous primary channels. Within heterogeneous channels, there should exist more feasible channel-hopping sequences to explore channel availability for CRUs.

Furthermore, it is assumed in most sensing-based and probability-based protocols that the CRUs must be synchronized with the PUs and sense either the idle or busy state of PUs. However, these two assumptions are considered impractical in realistic circumstances for the following reasons: 1) there is no communication between the primary and the CR networks, which makes global synchronization difficult; and 2) perfect spectrum sensing requires excessive sensing time, which degrades channel utilization especially for shorter slot times. In this paper, based on channelhopping schemes, we examine analytical models for both the probability of channel availability for the CRUs and the average frame delay of the PUs. It is noted that the CR networks with both the paired CRUs and arbitrary communications between the CRUs, i.e., the generalized CR network, are considered for establishing the analytical models. The proposed models incorporate possible collision events due to imperfect synchronization and sensing under a primary network containing multiple channels. Each channel is modeled as a Geo/G/1 queuing. system. By exploiting this realistically coexisting system, an optimal channel-hopping sequence (OCS) based on dynamic programming (DP) is derived in order to achieve maximum aggregate throughput for the CRUs and the average frame delay of the PUs with quality-of-service (QoS) guaranteed. Based on the proposed OCS scheme, optimal load balance can be achieved between the probability of channel availability and channel utilization within the CR network by knowing the frame arrival probabilities of PUs.
Moreover, the logical partition problem as described in [15] will occur in generalized CR networks, where the CR transmitter cannot find its corresponding $\mathrm{CR}$ receiver if the receiver switches to another channel in order to serve as a transmitter to conduct data delivery for another receiver. In such a case, severe degradation in aggregate throughput can happen due to the decreased probability in connectivity between the CRUs, especially under heavy CR traffic. Therefore, two algorithms, i.e., the wake-up successive contention (WSC) and the wake-up counter-reset successive contention (WCSC) mechanisms, are proposed to enhance the aggregate throughput of CRUs in generalized CR networks. These two schemes focus on how to alleviate the logical partition problem by increasing the number of negotiations from a blind spot in imperfect sensing and revising the contention mechanisms between the CRUs. Numerical results are presented to illustrate the performance of the proposed approaches in comparison with conventional schemes. It is clear that the proposed algorithms can capture the rapidly varying opportunities of spectrum holes for CRUs.

Noted that a preliminary design of the OCS scheme for paired CR networks was first presented in our previous work in [16]. A more comprehensive design and performance comparison for generalized CR networks will be conducted in this paper. The rest of this paper is organized as follows: The system models for both paired and generalized CR networks are presented in Section 2. Section 3 provides the throughput analysis in paired CR networks. Based on the analysis, the proposed OCS scheme is modeled and derived by dynamic programming. In Section 4, the proposed OCS algorithm associated with enhanced schemes are addressed under generalized CR networks. Section 5 illustrates performance evaluation for the proposed mechanisms; while the conclusions are drawn in Section 6.

\section{System Models}

In order to discuss the channel-hopping protocol for multiple CRUs, a simplified CR network with CRUs communicating in pairs will first be discussed. On the other hand, generalized network with CRUs arbitrarily communicating with each other will be addressed thereafter. This section will describe the system models including the network architecture, traffic model, and sensing model for the proposed OCS scheme. Section 2.1 explains those models under paired CR network; while those for generalized CR networks are addressed in Section 2.2.

\subsection{System Models for Paired CR Network}

\subsubsection{Network Architecture and Traffic Model}

The schematic diagram for coexistence of both the primary and CR networks is illustrated in Fig. 1. Noted that centralized downlink communication is considered for primary network while the CRUs in CR network communicate with each other based on distributed manner. The timing diagram of multichannel operations for these two networks is shown in Fig. 2. Both the primary and CR networks are slotted systems with the same slot duration $T_{s}$, where the time slots for $\mathrm{CR}$ network are depicted as the 


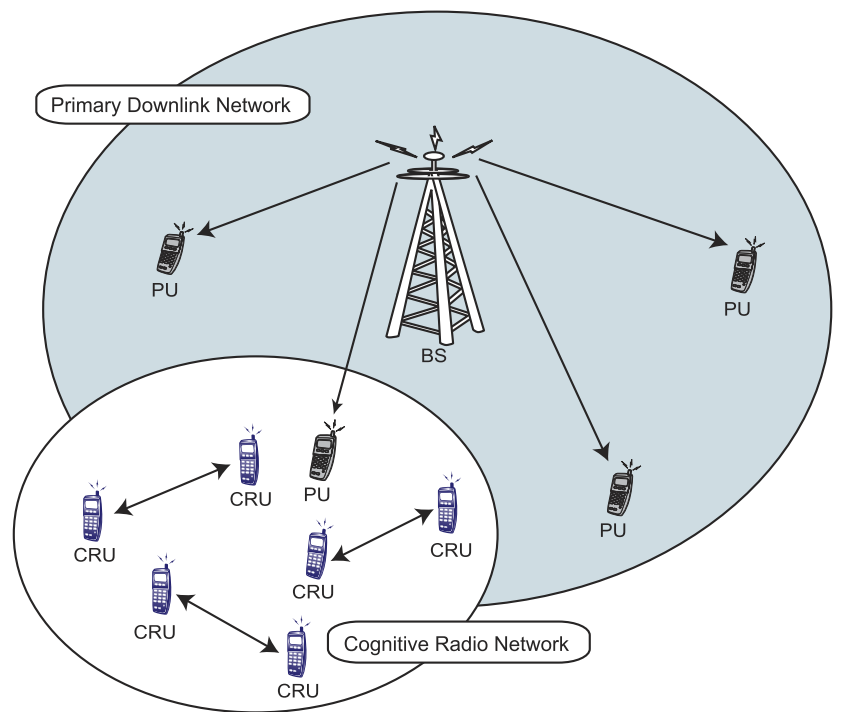

Fig. 1. Network scenarios for the coexistence of both the primary and the CR networks.

dotted vertical lines in Fig. 2. Since there is no communication between the PUs and CRUs, imperfect synchronization is considered where the time difference between these two networks can be observed at each starting epoch. For example, as shown in Fig. 2, the asynchronous time difference is obtained as $\Delta t=t_{2}-t_{1}$ where the $\mathrm{PU}$ in channel 2 starts transmitting the data at time $t_{1}$ and a CRU transmits its frame at $t_{2}$. Furthermore, the primary network has $M$ channels with identical bandwidth, and each channel is independently occupied by a PU with Bernoulli arrival process [17], [18] with the probability $\lambda_{i}$ of one frame arrival and the probability $1-\lambda_{i}$ of no frame arrival at the starting epochs for $i=1,2, \ldots, M$. Note that infinite queue capacity is assumed for each channel, where the frame duration is equal to a slot length as shown in Fig. 2.

Moreover, the proposed OCS scheme is designed based on PU's long term statistics. The assumption of known PU's traffic model is considered feasible since there are different organizations and companies concentrate on the establishment of traffic models in order to provide reasonable simulation assumptions for system performance comparisons. For example, two FTP traffic models have been constructed in the 3GPP [19] for performance evaluation of long term evolution (LTE) standard. Therefore, it is reasonable to assume that PU's traffic is known to CRUs. Moreover, there also exists a great amount of research studies for CR networks that adopt the same assumption of known PU's traffic pattern, e.g., [6], [7], [8], [9], [11], [12], [17]. The service length of each PU can be determined given that the PU will retransmit data until success, and the derivation will be presented in Section 3.1.

The single-hop CR network contains $N_{p}$ pairs of CRUs (CRPs) where each pair is consisted of two CRUs including a transmitter and a receiver. Since centralized downlink communication is adopted for primary network, it is reasonable to consider single-hop scenario that all CRPs in $\mathrm{CR}$ network are communicating based on distributed manner. Furthermore, multihop transmissions within a CRP, i.e., with the existence of intermediate relaying nodes,

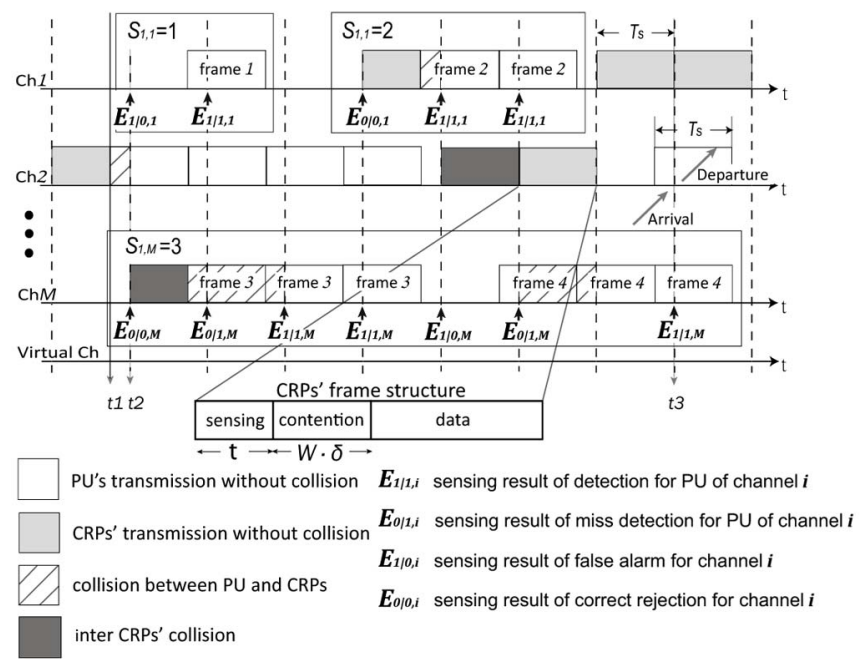

Fig. 2. Timing diagram for the coexistence of both the primary and the CR networks.

is not considered in this paper due to its difficulties on both implementation and analysis. Moreover, saturation traffic is considered for data transmission of CRPs within the network. The reason is that the worst case that affects PU's QoS requirement can therefore be examined with saturation traffic from CRPs. Meanwhile, it can be utilized to observe the maximum transmission capacity that is allowed for each CRP in the network.

It is also considered that data communication only happens within a CRP and all CRPs are affected by the same set of PUs. As mentioned at the beginning of this section, centralized downlink communication is adopted for primary network. Therefore, every CRU will be impacted by the same set of PUs since all PU's data transmissions are originated from a centralized base station. Based on the channel-hopping protocol as in [11], each CRP has to follow a default channel-hopping sequence generated by a pseudo random generator with the same discrete probability distribution $\mathbf{P}$, which is defined as $\mathbf{P}=\left[p_{1}, \ldots, p_{M}, p_{v i r}\right]$ with $p_{i}$ denoting the channel-hopping probability for $i=1,2, \ldots, M$. The distribution $\mathbf{P}$ is utilized to stochastically determine which channel to hop to, and to carry out sensing and contending for data transmission in a reasonably fair manner. In other words, the design of $\mathbf{P}$ corresponds to the design of a channel-hopping sequence. The frame structure of CRPs including sensing, contending, and data transmission phases is illustrated in Fig. 2. Moreover, the virtual channel that accounts for the relinquishment in transmissions of certain CRPs is represented as $p_{v i r}=1-\sum_{i=1}^{M} p_{i}$.

\subsubsection{Sensing Model}

In realistic situation, sensing decisions of CRPs is considered imperfect. In order to represent the effects from imperfect sensing, two probabilities are considered as follows: 1) the detection probability $p_{d}$ for detecting the PU when the PU does exist, and 2) the probability of false alarm $p_{f a}$ for detecting the PU while the PU does not exist. The relationship between $p_{d}$ and $p_{f a}$ has been studied in [20] for an energy detector, which can be obtained for each CRP as 


$$
p_{f a}=Q\left(\sqrt{2 \gamma+1} Q^{-1}\left(p_{d}\right)+\sqrt{\tau f_{s}} \gamma\right),
$$

where $\tau$ is the sensing time, $\gamma$ is PU's signal-to-noise ratio (SNR) acquired at the CRP's receiver, and $f_{s}$ denotes the channel sampling rate of the CRP. The $Q(\cdot)$ function represents the complementary distribution function of a standard Gaussian variable. Noted that detection probability $p_{d}$ is also called the sensing threshold which can be adjusted by CRP, and $p_{f a}$ is an increasing function of $p_{d}$ under fixed values of $\tau, \gamma$, and $f_{s}$. Moreover, it is reasonable to assume that the detection probabilities as well as false alarm probabilities of all CRPs are independent under realistic environment. The reason is that incorrect decisions, i.e., false alarm and misdetection, are in general background noises, which is considered feasible to be assumed as i.i.d. background noise for each CRP. On the other hand, the detection probabilities $p_{d}$ of all CRPs are conditional on all CRPs with the same SNR value, which is employed to let all CRPs have the same sensing behavior.

According to (1), it can be found that significant amount of sensing time $\tau$ is required for achieving perfect sensing, i.e., to exactly sense the state of PU to be either busy $\left(p_{d}=1\right)$ or idle $\left(p_{f a}=0\right)$. The required time for perfect sensing is considered much larger than the slot duration $T_{s}=10 \mathrm{~ms}$ as specified in IEEE 802.22 standard [3], [21] owing to the limitation in hardware and sensing algorithms. Therefore, perfect sensing is considered impractical and will severely degrade channel utilization. Furthermore, by assuming all CRPs with the same sensing threshold $p_{d}$, the equivalent detection probability can be written as $p_{d}^{n}$ given that $n$ out of $N_{p}$ CRPs hopping to a specific channel. The reason is that PU's data will not be collided by CR transmitters only if all the CR transmitters can correctly detect the signals from PU. Note that $n$ CRPs indicate that there are $n$ transmitters and $n$ receivers in paired CR networks. The effective false alarm probability $p_{f a}^{n}$ for $n$ out of $N_{p}$ CRPs hopping to a channel can also be computed in the similar manner. Hence, the average detection probability $P_{D, i}$ for CRPs hopping to channel $i$ all with correct detection and the average false alarm probability $P_{F, i}$ for CRPs hopping to channel $i$ all with false alarm can be, respectively, written as

$$
\begin{gathered}
P_{D, i}=\sum_{n=0}^{N_{p}} H_{N_{p}, n, i} p_{d}^{n}=\left[1-p_{i}\left(1-p_{d}\right)\right]^{N_{p}}, \\
P_{F, i}=\sum_{n=0}^{N_{p}} H_{N_{p}, n, i} p_{f a}^{n}=\left[1-p_{i}\left(1-p_{f a}\right)\right]^{N_{p}},
\end{gathered}
$$

where $H_{N_{p}, n, i}$ represents the probability of $n$ out of the $N_{p}$ CRPs hopping to channel $i$ with probability $p_{i}$ as

$$
H_{N_{p}, n, i}=\left(\begin{array}{c}
N_{p} \\
n
\end{array}\right) p_{i}^{n}\left(1-p_{i}\right)^{N_{p}-n} .
$$

Note that the detection probability $P_{D, i}$ and false alarm probability $P_{F, i}$ in (2a) and (2b) are defined to be average values considering that the number of CRPs hopping to a channel can range from 0 to $N_{p}$. In fact, detection probabilities in all channels are correlated, so are false alarm probabilities. However, all $P_{D, i}$ and $P_{F, i} \forall i$ are approximated to be independent in (2) in order to reduce derivation complexity such that the design of channelhopping sequence can be implemented. Based on the probabilities $P_{D, i}$ and $P_{F, i}$ as derived in (2a) and (2b), the probability of channel availability, average frame delay of PUs, and aggregate throughput in paired CR network will be obtained in the next section.

\subsection{System Models for Generalized CR Networks}

\subsubsection{Network Architecture and Traffic Model}

The CR network with CRUs in pairs for communication has been addressed in the previous section. However, the CRUs are not always transmitting in pairs since they may have data to be delivered to different CRUs in the network. In generic scenarios, the CRUs can be classified into either the transmitting group when they have data to deliver or the nontransmitting group if they have no data to transmit. The traffic of each CRU is considered to be Bernoulli arrival with the probability $\lambda_{C R}$ of one frame arrival and the probability $1-\lambda_{C R}$ of no frame arrival at the starting epochs as in [22]. Note that it is not feasible to consider saturation traffic for CRUs in generalized networks since there will be no $C R$ receiver to accept data from those CRUs in the transmitting group. Furthermore, no queue capacity is considered for each CRU and every CRU has equal probability in transmission with other CRUs.

\subsubsection{Sensing Model}

As was presented in Section 2.1.2 for paired CR networks, the problem resulting from imperfect sensing will also be addressed in generalized CR networks. The average detection probability $P_{D, i}$ and average false alarm probability $P_{F, i}$ for CRUs as stated in (2a) and (2b), respectively, will be recomputed under generalized CR networks, which are denoted as $\tilde{P}_{D, i}$ and $\tilde{P}_{F, i}$. The main feature of generalized CR networks is that there is comparably smaller probability for the transmitter and its corresponding receiver to have the same hopping sequences under either large number of channels or large number of CRUs. Consider that all CRUs are with the same sensing threshold $p_{d}, \tilde{P}_{D, i}$ represents the average detection probability for CRUs in the transmitting group hopping to channel $i$ all with correct detection; while $\tilde{P}_{F, i}$ denotes the average false alarm probability for CRUs in the transmitting group hopping to channel $i$ all with false alarm. Therefore, $\tilde{P}_{D, i}$ and $\tilde{P}_{F, i}$ can be, respectively, obtained as

$$
\begin{aligned}
\tilde{P}_{D, i}= & \sum_{n=0}^{N_{u}} \sum_{y=0}^{n}\left(\begin{array}{c}
N_{u} \\
n
\end{array}\right) \lambda_{C R}^{n}\left(1-\lambda_{C R}\right)^{N_{u}-n} \\
& \cdot\left(\begin{array}{c}
n \\
y
\end{array}\right) p_{i}^{y}\left(1-p_{i}\right)^{n-y} \cdot p_{d}^{y} \\
= & \sum_{n=0}^{N_{u}} \sum_{y=0}^{n} H_{n, y, i} T_{N_{u}, n} p_{d}^{y} \\
= & {\left[1-\lambda_{C R}+\lambda_{C R}\left(1-p_{i}+p_{i} p_{d}\right)\right]^{N_{u}}, } \\
\tilde{P}_{F, i}= & \sum_{n=0}^{N_{u}} \sum_{y=0}^{n} H_{n, y, i} T_{N_{u}, n} p_{f a}^{y} \\
= & {\left[1-\lambda_{C R}+\lambda_{C R}\left(1-p_{i}+p_{i} p_{f a}\right)\right]^{N_{u}}, }
\end{aligned}
$$


where $N_{u}$ denotes the total number of CRUs in the network. The parameter $H_{n, y, i}$ is defined similar to (3) indicating the probability of $y$ out of $n$ CRUs hopping to channel $i$ with probability $p_{i}$. Note that both $\tilde{P}_{D, i}$ and $\tilde{P}_{F, i} \forall i$ are also approximated to be independent. Moreover, $T_{N_{u}, n}$ represents the probability of $n$ out of $N_{u}$ CRUs possess data for delivery with transmitting probability $\lambda_{C R}$ as

$$
T_{N_{u}, n}=\left(\begin{array}{c}
N_{u} \\
n
\end{array}\right) \lambda_{C R}^{n}\left(1-\lambda_{C R}\right)^{N_{u}-n}
$$

Note that both $\tilde{P}_{D, i}$ and $\tilde{P}_{F, i}$ are defined to be average values considering that the number of CRUs hopping to a channel can range from 0 to $N_{u}$, and each CRU possesses with the transmitting probability $\lambda_{C R}$ for data delivery.

\section{Proposed Optimal Channel-Hopping Sequence Approach under Paired CR NETWORKS}

In this section, the proposed OCS scheme will be derived under paired $C R$ networks. The probability of channel availability for CRPs and the PU's average frame delay will first be computed in Section 3.1, which result in the derivation of CRPs' aggregate throughput in Section 3.2. The formulation of proposed OCS approach and its corresponding DP scheme will be addressed in Sections 3.3 and 3.4, respectively.

\subsection{Probability of Channel Availability for CRPs and PU's Average Frame Delay}

In this section, the impacts caused by imperfect synchronization and sensing of CRPs are analyzed for the probability of channel availability and average frame delay of primary queuing network. The primary network in each channel is a Geo/G/1 discrete-time queuing system with retransmitting capability if its frames are collided by CRPs. Noted that the arrivals and departures, i.e., the service completions, of primary network should occur at the starting epochs simultaneously. However, this type of system is considered intractable for analyzing the average system size. In order to ensure the analysis to be tractable, the arrival first (AF) scheme [23] is introduced as the scheduling policy for the queue. In other words, the frame arrivals take precedence over departures at the starting epochs, e.g., at time $t_{3}$ in channel 2 as shown in Fig. 2. It has been proven in [23] that the distribution of system size observed at the departure points by adopting the AF scheme will be identical to that of the original Geo/G/1 system. Therefore, the probability of channel availability for CRPs and the average frame delay of PU can be analyzed based on the derivation of system size distribution [24], [25] at the departure points.

Considering channel $i$ for $i=1,2, \ldots, M$, let $X_{m, i}$ be defined as the discrete random variables (RVs) of the number of PU's frames waiting for transmission observed at the $m$ th departure point. $S_{1, i}$ and $S_{2, i}$ are denoted as the RVs of service time (or delay) of one PU's frame under the condition that $X_{m, i}=0$ and $X_{m, i}>0$, respectively. Note that both $S_{1, i}$ and $S_{2, i}$ are independent of $m . A_{1, i}$ and $A_{2, i}$ are defined as the RVs of the number of PU's frames who arrive during the service time $S_{1, i}$ and $S_{2, i}$, respectively. The relationship between $X_{m, i}, A_{1, i}$, and $A_{2, i}$ can be obtained as

$$
X_{m+1, i}= \begin{cases}A_{1, i} & X_{m, i}=0 \\ X_{m, i}+A_{2, i}-1 & X_{m, i} \geq 1\end{cases}
$$

Consequently, the probability mass function (PMF) $a_{r, k, i}=$ $\operatorname{Pr}\left(A_{r, i}=k\right)$ for the number of arrival frames $A_{r, i}$ with $r=1$ and 2 can be obtained as

$$
\begin{aligned}
a_{r, k, i} & =\sum_{l=k}^{\infty} \operatorname{Pr}\left(S_{r, i}=l\right) \operatorname{Pr}\left(A_{r, i}=k \mid S_{r, i}=l\right) \\
& =\sum_{l=k}^{\infty}\left(\begin{array}{l}
l \\
k
\end{array}\right) \operatorname{Pr}\left(S_{r, i}=l\right) \lambda_{i}^{k}\left(1-\lambda_{i}\right)^{l-k} .
\end{aligned}
$$

Fig. 2 depicts the relationship between $S_{1, i}$ and CRPs' sensing results by considering the cases of $S_{1, i}=1,2$, and 3 . For channel $i$, let $\mathbf{E}_{\mathbf{1} \mid \mathbf{1}, \mathbf{i}}, \mathbf{E}_{\mathbf{0} \mid \mathbf{1}, \mathbf{i}}, \mathbf{E}_{\mathbf{1} \mid \mathbf{0}, \mathbf{i}}$, and $\mathbf{E}_{\mathbf{0} \mid \mathbf{0 , \mathbf { i }}}$ be, respectively, denoted as the sensing results of detection for PU, misdetection for PU, false alarm, and correct rejection. Hence, it can be intuitively observed that $P_{r}\left(\mathbf{E}_{\mathbf{1} \mid \mathbf{1}, \mathbf{i}}\right)=P_{D, i}, P_{r}\left(\mathbf{E}_{\mathbf{0} \mid \mathbf{1}, \mathbf{i}}\right)=1-P_{D, i}, P_{r}\left(\mathbf{E}_{\mathbf{1} \mid \mathbf{0}, \mathbf{i}}\right)=P_{F, i}$, and $P_{r}\left(\mathbf{E}_{\mathbf{0} \mid \mathbf{0}, \mathbf{i}}\right)=1-P_{F, i}$. Noted that $S_{1, i}$ also indicates the service time under the condition that there does not exist PU's frame in the previous slot according to PU's clock. Therefore, it can be observed that the sensing result made by CRPs in the previous slot becomes either $\mathbf{E}_{\mathbf{1} \mid 0, \mathrm{i}}$ or $\mathbf{E}_{\mathbf{0} \mid \mathbf{0}, \mathrm{i}}$, instead of either $\mathbf{E}_{1 \mid 1, i}$ or $\mathbf{E}_{0 \mid 1, i}$. As in Fig. 2, it can be seen that the successful frame transmitted by the PU requires two CRPs' successive $\mathbf{E}_{\mathbf{1} \mid \mathbf{1}, \mathbf{i}}$ except for $S_{1, i}=1$. Consequently, both the two cases $S_{1, i}=1$ and $S_{1, i}>1$ should be considered in the analysis. Moreover, the first sensing probability of either $P_{F, i}$ or $1-P_{F, i}$ condition on $S_{1, i}>1$ needs to be studied since the second sensing result should be $\mathbf{E}_{\mathbf{0} \mid \mathbf{1}, \mathbf{i}}$ if the first sensing result is $\mathbf{E}_{\mathbf{1} \mid 0, \mathbf{i}}$. Noted that $S_{1, i}=1$ represents the first sensing result as $\mathbf{E}_{\mathbf{1} \mid \mathbf{0}, \mathbf{i}}$ and the second sensing result to be $\mathbf{E}_{1 \mid 1, i}$. Furthermore, the SNR of CRPs is considered higher than that of PUs owing to the shorter communication range in CR networks. The carrier sensing before the contention phase cannot detect the existence of PU due to the different natures of spectrum sensing in the detector [26]. Let $c_{1, i}(z)$ and $q_{i}(z)$ be, respectively, defined as the $z$-transform of the PMF of $S_{1, i}$ and the PMF of transmitting until the existence of two successive $\mathbf{E}_{1 \mid 1, i}$. According to (2a) and (2b), $c_{1, i}(z)$ can be written as

$$
c_{1, i}(z)=P_{F, i}\left[P_{D, i} z+\left(1-P_{D, i}\right) q_{i}(z) z\right]+\left(1-P_{F, i}\right) q_{i}(z),
$$

and $q_{i}(z)$ is derived by recursive method as

$$
q_{i}(z)=f_{i}(z)\left[P_{D, i} z+\left(1-P_{D, i}\right) q_{i}(z) z\right],
$$

which can further be reorganized as

$$
q_{i}(z)=\frac{P_{D, i} f_{i}(z) z}{1-\left(1-P_{D, i}\right) f_{i}(z) z},
$$

where $f_{i}(z)$ represents the $z$-transform of geometric distribution with parameter $P_{D, i}$ as

$$
f_{i}(z)=\frac{P_{D, i} z}{1-\left(1-P_{D, i}\right) z} .
$$


Furthermore, $S_{2, i}$ denotes the service time for a new frame, i.e., not a retransmitted frame, that is served right after the previous frame. It indicates that the sensing result of CRPS in the last slot must be $\mathbf{E}_{1 \mid 1, i}$ such that the previous frame can leave the queue. The $z$-transform of the PMF of $S_{2, i}$ can be derived similar to $S_{1, i}$ as

$$
c_{2, i}(z)=P_{D, i} z+\left(1-P_{D, i}\right) q_{i}(z) z,
$$

where $q_{i}(z)$ is obtained from (10). Let $h_{r, i}(z)$ be defined as the $z$-transform of $a_{r, k, i}$ in (8) for $r=1$ and 2, the following relationship between $h_{r, i}(z)$ and $c_{r, i}(z)$ can be obtained:

$$
\begin{aligned}
h_{r, i}(z) & =\sum_{k=0}^{\infty} a_{r, k, i} z^{k}=\sum_{l=0}^{\infty} \operatorname{Pr}\left(S_{r, i}=l\right)\left(1-\lambda_{i}+\lambda_{i} z\right)^{l} \\
& =c_{r, i}\left(1-\lambda_{i}+\lambda_{i} z\right) .
\end{aligned}
$$

Moreover, let $\pi_{j, i}$ be defined as the steady state probability of $X_{m, i}=j$, i.e., with $m \rightarrow \infty$. The value of $\pi_{j, i}$ corresponds to the steady state system size observed at the departure points, which can be acquired as

$$
\pi_{j, i}=\pi_{0, i} a_{1, j, i}+\sum_{k=1}^{j+1} \pi_{k, i} a_{2, j-k+1, i} .
$$

Based on (13), the $z$-transform of $\pi_{j, i}$, denoted as $g_{i}(z)$, can be derived as

$$
g_{i}(z)=\frac{\pi_{0, i}\left[h_{1, i}(z) z-h_{2, i}(z)\right]}{z-h_{2, i}(z)} .
$$

Therefore, according to the boundary conditions $g_{i}(1)=1$, $h_{1, i}(1)=1$, and $h_{2, i}(1)=1$, the probability of channel availability for CRPs in the $i$ th channel can be obtained by using the L'Hopital's rule as

$$
\pi_{0, i}=\left.\frac{1-h_{2, i}^{\prime}(z)}{h_{1, i}^{\prime}(z) z+h_{1, i}(z)-h_{2, i}^{\prime}(z)}\right|_{z=1},
$$

where $h_{r, i}^{\prime}$ denotes the derivative of $h_{r, i}$ for $r=1$ and 2. The average frame delay of PU in the $i$ th channel can be derived by adopting the Little's Theorem as

$$
D_{i}\left(p_{i}\right)=L_{i}\left(p_{i}\right) / \lambda_{i},
$$

where the average system size for the $i$ th channel $L_{i}\left(p_{i}\right)$ is acquired by taking the derivative of (15) at $z=1$ using the L'Hopital's rule as

$$
L_{i}\left(p_{i}\right)=\sum_{j=0}^{\infty} j \pi_{j, i}=\left.g_{i}^{\prime}(z)\right|_{z=1} .
$$

Noted that the probability of channel availability for CRPS $\pi_{0, i}$ in (16) will be utilized in the computation of system throughput in next section. The average frame delay of the PU $D_{i}\left(p_{i}\right)$ in (17) will be adopted as a major constraint of proposed optimization problem in Section 3.3.

\subsection{Aggregate Throughput of CRPs}

Based on the analysis in primary network under certain channel-hopping sequence of CRPs, the aggregate throughput of CRPs can be obtained according to CRP's frame structure as shown in Fig. 2. With the CRPs transmitting in a single-hop wireless network, there are $n$ out of $N_{p}$ CRPs hopping to the $i$ th channel with the probability $H_{N_{p}, n, i}$ as defined in (3). Due to imperfect sensing, there is probability of $\alpha$ out of $n$ CRPs with correct sensing while channel $i$ is idle as

$$
F_{n, \alpha}=\left(\begin{array}{l}
n \\
\alpha
\end{array}\right)\left(1-p_{f a}\right)^{\alpha} p_{f a}^{n-\alpha}
$$

After sensing the channel availability, the contention-based scheme as defined in IEEE 802.11 distributed coordination function (DCF) mode [27] will be adopted, which consists of two-way handshakes including data transmission and acknowledgement. The $\alpha$ CRPs who sense the channel idle will have the privilege to contend for data transmission. Each CRP transmitter will wait for a randomly chosen backoff value in the interval of $[0, W-1]$ before data delivery, where $W$ denotes a fixed window size. The CRPs can transmit data only if their backoff values count down to zero, and they will stop the backoff process if data transmission from other CRP has been observed while listening. Noted that exponential backoff and request-tosend/clear-to-send (RTS/CTS) exchanges are not adopted in this approach since small frame size of CRP is considered. The reason of using smaller CRP's frame size is due to the requirements for frequent channel sensing in order to serve for highly time-varying CR traffic. Moreover, the slot size $T_{s}$ is in general selected with small values [3], [28] according to the underlying CR specifications. Consequently, it is reasonable to allow only a single frame transmission in each time slot. Therefore, the average successful transmission time $C_{\alpha}$ with no intercollision between $\alpha$ CRPs can be obtained as

$$
C_{\alpha}=\frac{\alpha}{W} \sum_{\beta=1}^{W}\left[T_{s}-(\beta-1) \delta-\tau\right]\left(1-\frac{\beta}{W}\right)^{\alpha-1},
$$

where $\delta$ denotes the minislot for carrier sensing. The term $\alpha \cdot(1-\beta / W)^{\alpha-1}$ in (20) denotes the probability for successful data transmission in the $\beta$ th minislot. $\left[T_{s}-(\beta-1) \delta-\tau\right]$ indicates the corresponding data transmission time, where $(\beta-1) \delta$ represents the waiting time for random backoff. Based on (3), (16), (19), and (20), the throughput of CRPs in the $i$ th channel $\eta_{i}\left(p_{i}\right)$ can be acquired as

$$
\eta_{i}\left(p_{i}\right)=\frac{1}{T_{s}} \pi_{0, i}\left(1-\lambda_{i}\right) \sum_{n=1}^{N_{p}} \sum_{\alpha=1}^{n} H_{N_{p}, n, i} F_{n, \alpha} C_{\alpha},
$$

where the unit of throughput is in time slots. The throughput of CRPs in the $i$ th channel $\eta_{i}\left(p_{i}\right)$ in (21) scaled with normalization factor $1 / T_{s}$ is composed by the probability of channel availability $\pi_{0, i}$ in (16) and the probability of $\left(1-\lambda_{i}\right)$ that no PU is conducting data transmission while a CRP is successfully transmitting. Moreover, the term $\sum_{n=1}^{N_{p}} \sum_{\alpha=1}^{n} H_{N_{p}, n, i} F_{n, \alpha} C_{\alpha}$ in (21) denotes average throughput of the $i$ th channel on condition that CRPs are with correct channel sensing. As a result, the aggregate throughput for all $M$ channels with probability distribution $\mathbf{P}$ can be obtained as 


$$
\begin{aligned}
\eta(\mathbf{P})= & \sum_{i=1}^{M} \eta_{i}\left(p_{i}\right)=\frac{1}{T_{s}} \sum_{i=1}^{M} \pi_{0, i}\left(1-\lambda_{i}\right)\left[\frac{N_{p} p_{i}\left(1-p_{f a}\right)}{W}\right] \\
& \cdot \sum_{\beta=1}^{W}\left[T_{s}-(\beta-1) \delta-\tau\right]\left[1-\frac{\beta p_{i}\left(1-p_{f a}\right)}{W}\right]^{N_{p}-1} .
\end{aligned}
$$

In the next section, the aggregate throughput $\eta(\mathbf{P})$ derived in (22) will be utilized as the optimization criterion for proposed OCS scheme.

\subsection{Proposed OCS Approach}

In previous section, throughput analysis is conducted under realistic circumstances with the coexistence of PUs and CRPs. In this section, an approach for obtaining OCS will be developed in order to maximize aggregate throughput of CRPs in (22) under the QoS constraints of PUs. The optimization problem for OCS can be formulated as

$$
\begin{aligned}
& \quad \mathbf{P}^{*}=\arg \max _{\mathbf{P}} \eta(\mathbf{P}) \\
& \text { s.t. } \quad D_{\min } \leq D_{i}\left(p_{i}\right) \leq D_{c, i}, \quad 0 \leq p_{i} \leq 1, \\
& \quad \sum_{i=1}^{M} p_{i} \leq 1, \text { for } i=1,2, \ldots, M,
\end{aligned}
$$

where $\mathbf{P}^{*}=\left[p_{1}^{*}, \ldots, p_{M}^{*}, p_{v i r}^{*}\right]$ represents the corresponding OCS with $p_{i}^{*}$ denoting the optimal channel-hopping probability. The average frame delay of PU in the $i$ th channel $D_{i}\left(p_{i}\right)$ is obtained from (17). The parameter $D_{c, i}$ indicates the delay constraint for QoS requirement of PU in channel $i$, and $D_{\min }$ corresponds to a slot size if there is no collision within PU's transmissions. In other words, the emphasis of this paper is to obtain OCS within $N_{p}$ CRPs for optimal load balance considering PU's QoS requirements.

Intuitively, the optimization problem in (23) can be viewed as a sequential optimal decision problem from channel 1 to $M$. However, due to nonlinear relationship between throughput at the $i$ th channel $\eta_{i}\left(p_{i}\right)$ in (21) and the probability $p_{i}$ for $i=1$ to $M$, each decision on hopping probability $p_{i}$ cannot be independently determined since the throughput will be influenced by the rest of undecided channels. In other words, it is not possible to directly allocate 100 percent hopping probability into a channel with the lowest frame arrival probability since potential throughput may exist in the remaining channels that are unassigned with hopping probabilities. In order to resolve this problem, the DP-based approach in [29] is utilized for obtaining the OCS as will be shown in next section.

\subsection{Dynamic Programming Formulation for Proposed OCS Approach}

In this section, the optimization problem in (23) for multiple channel operations can be formulated into a DP problem based on [29]. A reward function $\phi_{i}\left(\epsilon_{i}\right)$ for channel $i$ denotes the maximum throughput summed from channel $i$ to channel $M$ with channel available probability $\epsilon_{i}$, which indicates the probability to be allocated from channel $i$ to $M$. Moreover, an instant reward function is defined to be $\eta_{i}\left(p_{i}\right)$ as in (21) plus the constraint $p_{c, i}$ on channel-hopping probability $p_{i}$. Noted that the constraint $p_{c, i}$ is a replacement of delay constraint $D_{c, i}$ since the average frame delay $D_{i}\left(p_{i}\right)$ in (17) is a strictly increasing function, i.e., one-to-one mapping, of channel-hopping probability $p_{i}$. The DP recursive form for proposed OCS scheme can therefore be written as

$$
\phi_{i}\left(\epsilon_{i}\right)=\max _{0 \leq p_{i} \leq \min \left\{p_{c, i}, \epsilon_{i}\right\}}\left\{\eta_{i}\left(p_{i}\right)+\phi_{i+1}\left(\epsilon_{i}-p_{i}\right)\right\},
$$

where $\epsilon_{1}=1$ and $\epsilon_{i+1}=\epsilon_{i}-p_{i}$. The maximum aggregate throughput in OCS can be obtained as

$$
\eta\left(\mathbf{P}^{*}\right)=\phi_{1}\left(\epsilon_{1}\right)
$$

where $\mathbf{P}^{*}$ from the OCS approach is the combination of channel-hopping probabilities acquired by the DP recursion in each channel. The allowable channel-hopping probability $p_{i}$ for each channel is quantized from 0 to 1 with quantization level $\Delta_{p}$. The selection of $\Delta_{p}$ is determined based on the quantization level in power as depicted in [30], which will relate to the accuracy of proposed OCS approach. First of all, the original problem can be partitioned into $\frac{1}{\Delta_{p}}+1$ subproblems by assigning $p_{1}=0$, $p_{1}=\triangle_{p}, p_{1}=2 \triangle_{p}, \ldots, p_{1}=1$, and the remaining corresponding unassigned probabilities becomes $1,1-\triangle_{p}$, $1-2 \triangle_{p}, \ldots, 0$. For example, the first subproblem of (24) can be written as

$$
\begin{aligned}
\phi_{1}\left(\epsilon_{1}=1\right)= & \max \left\{\eta_{1}\left(p_{1}=0\right)+\phi_{2}\left(\epsilon_{1}-\left(p_{1}=0\right)\right),\right. \\
& \eta_{1}\left(p_{1}=\triangle_{p}\right)+\phi_{2}\left(\epsilon_{1}-\left(p_{1}=\triangle_{p}\right)\right), \ldots, \\
& \left.\eta_{1}\left(p_{1}=1\right)+\phi_{2}\left(\epsilon_{1}-\left(p_{1}=1\right)\right)\right\} .
\end{aligned}
$$

Similarly, the remaining subproblems $\phi_{2}$ to $\phi_{M}$ can also be acquired by recursively breaking them into smaller subproblems until the probability $p_{M}$ is assigned. After these $\frac{1}{\Delta_{p}}+1$ subproblems are solved, the solution for original optimization problem in (24) can therefore be acquired. As indicated in [31], linear complexity of $O\left(M \cdot\left(1 / \Delta_{p}\right)^{2}\right)$ that is proportional to the number of channels $M$ can be obtained by adopting the DP formulation in (24) and (25) for proposed OCS approach. After offline solving the OCS for different numbers of CRPs, the proposed OCS scheme can consequently be recorded into a lookup table in order to acquire the optimal channel-hopping probability for $N$ CRPs in real-time implementation. As a result, under the same $\Delta_{p}$, the DP scheme for proposed OCS approach will be computationally efficient in finding the optimal hopping probability compared to the brute force scheme with exponential complexity of $O\left(\left(1 / \Delta_{p}\right)^{M}\right)$. Therefore, the computation time for executing DP can be considered a small and negligible overhead for CRPs to acquire updated channel-hopping sequence.

\section{Proposed Optimal Channel-Hopping Sequence Approach under Generalized CR NeTWORKS}

When CRUs are in the transmitting group, they have to follow the channel-hopping sequence of their corresponding receivers to conduct channel negotiations and data transmission. On the other hand, for those CRUs in the nontransmitting group, they only need to follow their own channel-hopping sequence to hop between channels. 
However, there is a severe problem that arises in generalized CR networks called logic partition problem as stated in [15]. The CR transmitter cannot find its corresponding CR receiver since the receiver may also be in the transmitting group, which can severely degrade the CR network throughput. This type of situations frequently occur under CR network with heavy traffic. In order to address the logic partition problem, the OCS approach for generalized CR network will be presented and the associated analysis of aggregate throughput will be provided in Section 4.1.

Furthermore, in paired CR networks, the events of fail transmission for CRPs are composed of intercollision among the CRPs and collision between PUs and CRPs. However, with the admission control and utilization of comparably larger contention window size in CR network, the probability of intercollision among CRPs can be considered insignificant. Therefore, it is unnecessary for all CRPs to contend successively by adopting the DCF mode in IEEE 802.11 standard. As described in Section 3.2, the two-way handshake process is utilized that the CRP who sense the channel idle can start the contention process and will terminate the backoff counter while observing the other CRP's transmissions. However, this type of simple negotiation will not be sufficient for generalized CR networks. The aggregate throughput for CR network will be severely degraded if the CR transmitter does not hop to the same channel as its corresponding CR receiver, especially in the network with heavy $C R$ traffic. In order to further alleviate this problem for throughput enhancement, both the WSC and the WCSC algorithms are proposed to be combined with the OCS approach in Sections 4.2 and 4.3.

\subsection{Aggregate Throughput and Proposed OCS Approach}

Based on $\tilde{P}_{D, i}$ in (4) and $\tilde{P}_{F, i}$ in (5), the probability of channel availability for CRUs and the average frame delay for PUs in generalized CR network can also be derived similar to the analysis as presented in Section 3.1, which are, respectively, denoted as $\tilde{\pi}_{0, i}$ and $\tilde{D}_{i}\left(p_{i}\right)$ for the $i$ th channel. The aggregate throughput for generalized CR network can therefore be computed in this section. Unlike the situations in paired CR networks, it is required to first obtain the rendezvous probability $p_{v, i}$ in generalized CR network which refers to the probability for a CR transmitter to find its intended CR receivers in the same channel $i$. It indicates that a CR transmitter can have the chance to communicate with its corresponding CR receiver. Under the assumption of equal probability for $\mathrm{CR}$ transmitters in choosing the receivers, the rendezvous probability $p_{v, i}$ at channel $i$ can be obtained as

$$
p_{v, i}=\frac{N_{u}-n}{N_{u}-1}+\frac{y_{i}-1}{N_{u}-1},
$$

with the total number of CRUs $N_{u}>1$. The parameter $n$ denotes the number of CRUs in the transmitting group, and $y_{i}$ represents the number of CRUs in the transmitting group that hop to the same channel $i$. The first term in (26) indicates the probability that the corresponding CR receiver of a CR transmitter exists in the nontransmitting group. On the other hand, the second term in (26) represents the probability that the corresponding $\mathrm{CR}$ receiver of a $\mathrm{CR}$ transmitter is in the transmitting group but hops to the same channel $i$ as the CR transmitter. Noted that $y_{i}=1$ indicates that only a single CR transmitter hops to the $i$ th channel. Similar to the derivation in Section 3.2, the throughput for channel $i$ can be obtained by combining $\tilde{\pi}_{0, i}$ and $p_{v, i}$ in (26) as

$$
\tilde{\eta}_{i}\left(p_{i}\right)=\frac{1}{T_{s}} \tilde{\pi}_{0, i}\left(1-\lambda_{i}\right) \sum_{n=1}^{N_{u}} \sum_{y=1}^{n} \sum_{\alpha=1}^{y} p_{v, i} H_{n, y, i} T_{N_{u}, n} F_{y, \alpha} C_{\alpha},
$$

for $N_{u}>1$, where $H_{n, y, i}, F_{y, \alpha}$, and $C_{\alpha}$ are obtained from (3), (19), and (20), respectively. Consequently, the aggregate throughput for generalized CR networks with the probability distribution $\mathbf{P}$ can be acquired as

$$
\begin{aligned}
\tilde{\eta}(\mathbf{P})= & \sum_{i=1}^{M} \tilde{\eta}_{i}\left(p_{i}\right)=\frac{1}{T_{s}} \sum_{i=1}^{M} \tilde{\pi}_{0, i}\left(1-\lambda_{i}\right)\left[\frac{N_{u} p_{i} \lambda_{C R}\left(1-p_{f a}\right)}{W}\right] \\
& \cdot \sum_{\beta=1}^{W}\left[1+p_{i} \lambda_{C R}-\lambda_{C R}-\frac{p_{i} \lambda_{C R} \beta\left(1-p_{f a}\right)}{W}\right] \\
& {\left[T_{s}-(\beta-1) \delta-\tau\right] } \\
& \cdot\left[\lambda_{C R}\left(1-\frac{\beta p_{i}\left(1-p_{f a}\right)}{W}\right)+1-\lambda_{C R}\right]^{N_{u}-2},
\end{aligned}
$$

for $N_{u}>1$. Intuitively, $\tilde{\eta}(\mathbf{P})=0$ for $N_{u}=1$. Similar to the derivations as stated in Section 3.3, the optimization problem to obtain the OCS $\tilde{\mathbf{P}}^{*}$ for generalized CR networks can be formulated similar to (23). Moreover, the DP formulation for OCS approach as described in Section 3.4 can also be applied for generalized CR networks.

\subsection{Enhancement with Wake-Up Successive Contention Algorithm}

In order to provide higher efficiency for generalized $\mathrm{CR}$ networks, the WSC scheme is proposed to increase the transmission opportunity for the contention mechanism. First of all, the four-way handshake RTS/CTS/DATA/ ACK as stated in [27] is used for communication between a CR transmitter and a CR receiver. In the original OCS approach, each CRU will only contend the channel once if its backoff counter reaches zero. In the WSC scheme, on the other hand, the CRUs will successively contend the channel until the end of contention phase if other CRUs' failed transmission has been observed. Noted that failed transmission of a CRU is indicated by the time duration of a single RTS frame plus a short interframe space (SIFS), which represents that a corresponding $C R$ receiver either cannot correctly receive the RTS frame or the receiver hops to the other channel.

Furthermore, due to the probability of false alarm caused by imperfect sensing, some CRUs will mistakenly think there exists a PU in the channel while the PU is not actively transmitting data in the channel. The CRUs will remain in the silent state without conducting channel contention and negotiation processes with their corresponding CR receivers. There will exist comparably less CRUs in a channel 


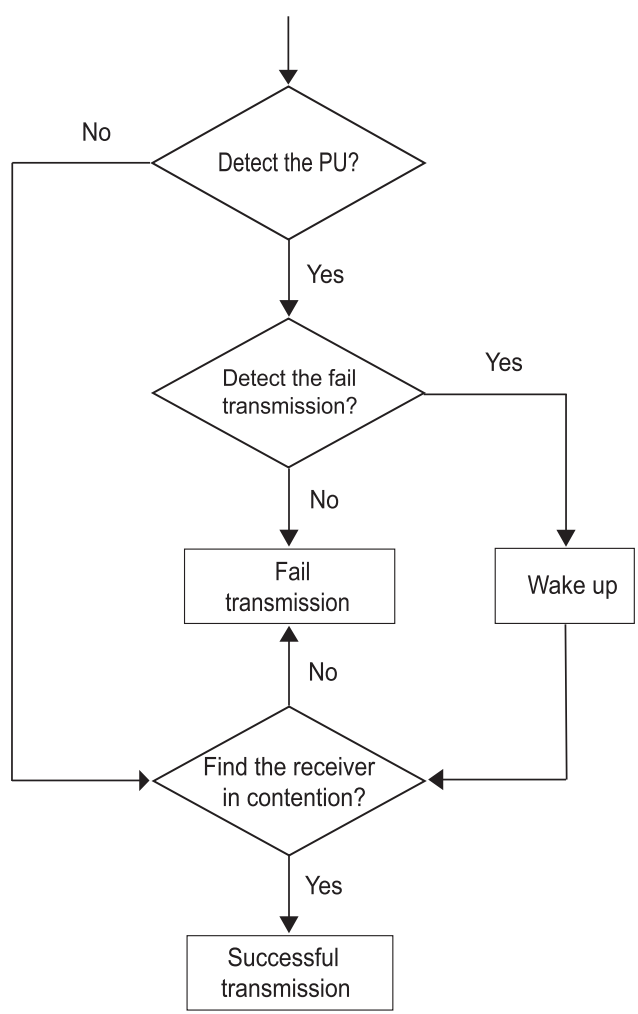

Fig. 3. Flow diagram of proposed WSC algorithm.

that are actively contending the channel which consequently decreases the transmission opportunities between CRUs. Therefore, as shown in Fig. 3, the wake-up mechanism in proposed WSC scheme is designed to increase the number of CRUs in the contention phase by allowing all CRUs who were blocked from accessing the channel into the contention phase while listening to the failed transmission of the other CRUs. Moreover, those wake-up CRUs will select their own backoff counter $C$ within the range of $[0, W-1]$. Noted that the CR transmitter that failed in its transmission will not successively join the afterwards contention process. In summary, the WSC algorithm is formed by the combination of fourway handshake, successive contention, and wake-up mechanism.

\subsection{Enhancement with Wake-Up Counter-Reset Successive Contention Algorithm}

The proposed WCSC scheme is designed based on the WSC algorithm by modifying the wake-up mechanism in each CRU. The design target of WCSC algorithm is to increase the number of negotiations between CRUs which consequently results in higher probability of successful transmissions. As in the WCSC scheme shown in Fig. 4, each time after the wake-up for channel contention, the CRU will reset its own backoff counter $C$ by randomly choosing a value in the range of $\left[W_{p}, W-1\right]$. The parameter $W_{p}=$ $\left\lceil\Delta t_{p} / \delta\right\rceil$ represents the number of minislot for the corresponding time interval $\Delta t_{p}$ that a CRU has encountered within the contention period. It can be observed that there can be more CRUs that possess the opportunity for channel contention compared to that in the WSC scheme which

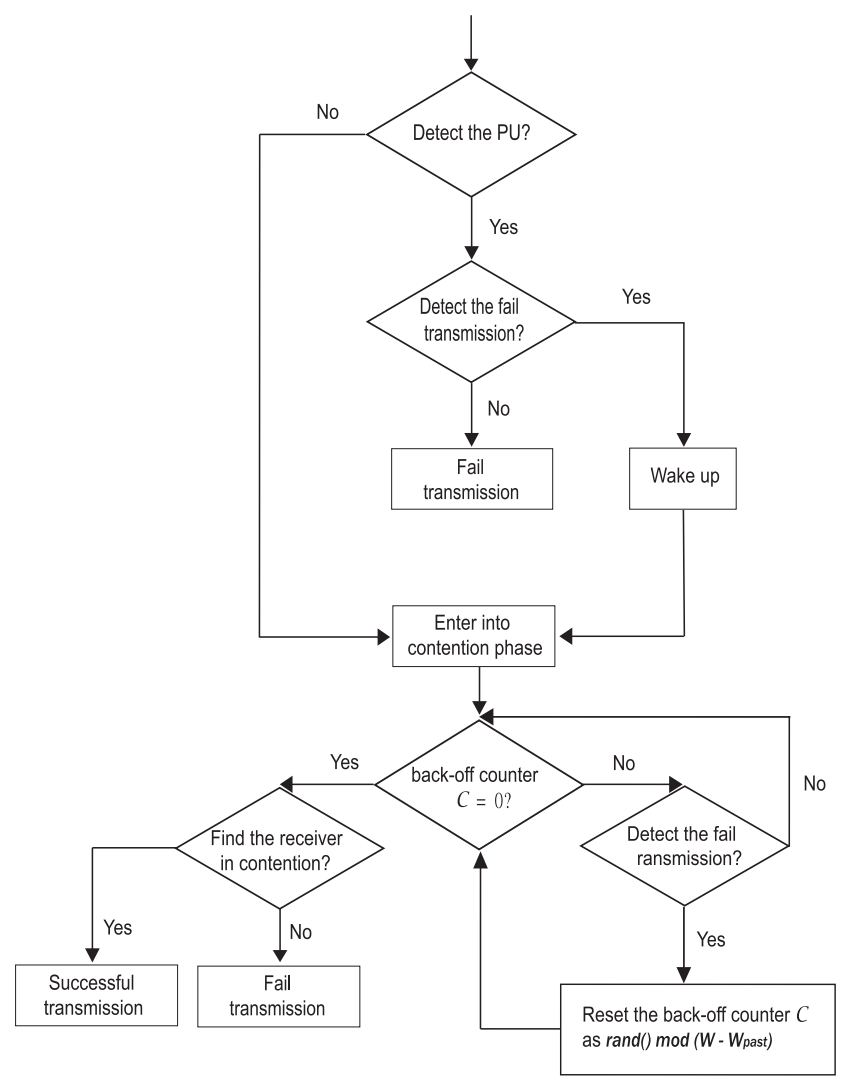

Fig. 4. Flow diagram of proposed WCSC algorithm.

utilizes fixed interval of backoff window in $[0, W-1]$. Noted that there is no guarantee for the proposed WCSC algorithm to outperform the WSC scheme. The reason is that there can be more collision happened with the existence of larger number of CRUs in a channel compared to the WSC scheme.

Moreover, these two enhancement schemes will not affect PU's average frame delay with reasons explained as follows: The major design concept of both schemes is that CRUs will successively contend the channel if other CRUs' failed transmission has been observed. Considering the case with the existence of PU in a channel, there can exist a CRU that misdetects the existence of PU and transmits its own data in a channel. Consequently, data from both PU and CRU will collide with each other and fail in transmission. According to the design of proposed schemes, other CRUs that listen to the occurrence of failed transmission will start to enter a channel and contend for data transmission in this frame duration. However, the PU's average frame delay will not be affected by the successive contention scheme since the PU's data have already been collided by the previous CRU that causes failed transmission. On the other hand, considering the case without the existence of PU in a channel, it is intuitive that no matter how many CRUs entering the channel will not affect the PU transmission behaviors, specifically on the average frame delay. Note that negotiations between CRUs are not required in order to recognize failed transmission since a CR transmitter can identify either the successful or failed transmission via listening to the existence of CTS 
TABLE 1

Simulation Parameters

\begin{tabular}{|l|l|l|l|}
\hline Primary network & Value & CR network & Value \\
\hline \hline Channel bandwidth $B$ & $6 \mathrm{MHz}$ & Over-sampling rate of CRUs $f_{s}$ & $8 B / 7$ \\
SNR of PU $\gamma$ & $-20 \mathrm{~dB}$ & Sensing time $\tau$ & $1 \mathrm{~ms}$ \\
Slot duration $T_{s}$ & $11.28 \mathrm{~ms}$ & Slot duration $T_{s}$ & $11.28 \mathrm{~ms}$ \\
& & Min-slot $\delta$ & $20 \mu \mathrm{s}$ \\
& & short interframe space (SIFS) & $10 \mu \mathrm{s}$ \\
& & Minimum channel bit rate & $2 \mathrm{Mbps}$ \\
& & RTS frame size & $160 \mathrm{bits}$ \\
& & CTS/ACK frame size & $112 \mathrm{bits}$ \\
& & Contention window size $W$ & 64 \\
& & Quantization level $\Delta_{p}$ & 0.001 \\
\hline
\end{tabular}

packet delivered by a CR receiver. Therefore, the proposed two enhanced schemes will not increase PU's average frame delay. This observation will further be validated in the performance evaluation section.

The performance of proposed WSC and WCSC schemes will be incorporated with the original OCS approach, i.e., denoted as OCS-WSC and OCS-WCSC schemes, respectively, and will be evaluated in the performance evaluation section under generalized CR networks. Noted that the major purpose for both the proposed OCS-WSC and OCSWCSC schemes is to increase the probability for the CR transmitter to find its corresponding $C R$ receiver in generalized $C R$ networks. Both enhancement can also be adopted for paired CR networks. However, it is intuitive to recognize that the overhead resulting from the four-way handshake can degrade the throughput performance for paired CR networks. It is considered sufficient to utilize the original OCS scheme for paired CR networks. On the other hand, in a network with both paired and nonpaired CRUs, the OCS-WSC and OCS-WCSC algorithms should be adopted which will treat each CRP as two individual CRUs. The negotiation between CRUs by adopting the four-way handshake and successive contention will be applied to all CRUs in order to effectively enhance the transmission probability and system throughput.

\section{Performance Evaluation}

In this section, theoretical analysis for proposed OCS approach will be validated via simulations under both paired and generalized CR networks. The performance of proposed OCS, OCS-WSC, and OCS-WCSC schemes will be evaluated and compared to the other conventional channelhopping sequences. Moreover, for finding the suboptimal solution of OCS, the discussion on quantization level $\Delta_{p}$ in the DP formulation will also be provided. Simulation parameters for both primary and CR networks are, respectively, listed in Table 1 . Noted that the parameters are adopted from the IEEE 802.22 standard in [3], [4] for CR network operating on TV bands; while those for the contention process are acquired from the IEEE 802.11 standard.

\subsection{Determination of Quantization Level in DP Formulation}

As described in Section 3.4, it is required to determine the quantization level $\Delta_{p}$ for DP formulation to obtain the suboptimal solution of OCS approach. Considering various values of quantized level $\Delta_{p}$, Figs. $5 \mathrm{a}$ and $5 \mathrm{~b}$ show the comparison for aggregate throughput of CRUs and average frame delay of PU, respectively, with respect to different numbers of CRUs. The number of channels is equal to $M=4$ where the PU's arrival rate for each channel is $\lambda_{i}=$ $0.1,0.1,0.3,0.3$ for $i=1,2,3,4$. Two cases of arrival rates for CRUs are considered as $\lambda_{C R}=0.4$ and 0.8 . The analytical results are obtained for aggregated throughput $\tilde{\eta}(\mathbf{P})$ and aggregate frame delay from Section 4.1 under generalized CR networks with sensing threshold $p_{d}=0.93$. The aggregate frame delay is the summation of average frame delay in each channel, i.e., $\tilde{D}=\sum_{i=1}^{M} \tilde{D}_{i}\left(p_{i}\right)$, which can be utilized as a measurement for the QoS requirement in primary system. Noted that the aggregate throughput $\tilde{\eta}(\mathbf{P})$ is normalized with slot duration $T_{s}$ for performance comparison.

It can be observed from both Figs. $5 a$ and $5 b$ that consistent suboptimal solution for OCS approach can be achieved with the selection of smaller value of quantization level $\Delta_{p}$. However, the granularity of quantization level $\Delta_{p}$ will be constrained by considering the hardware limitations and the computational complexity in DP approach. Therefore, according to Figs. $5 \mathrm{a}$ and $5 \mathrm{~b}$, the quantization level $\Delta_{p}$ for searching the OCS is set as 0.001 , which is accurate enough to obtain the proposed OCS in the simulation settings with reasonable number of CRUs and channels. In the following sections, $\Delta_{p}=0.001$ will be adopted for performance validation and comparison. 


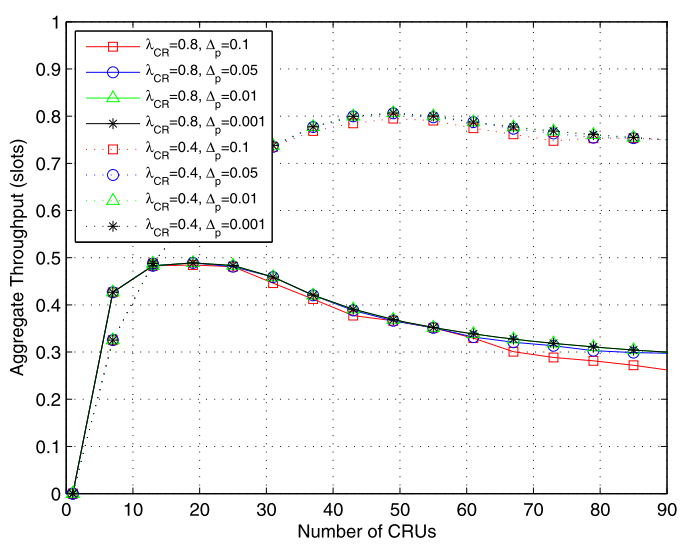

(a) Aggregate throughput of CRUs versus number of CRUs.

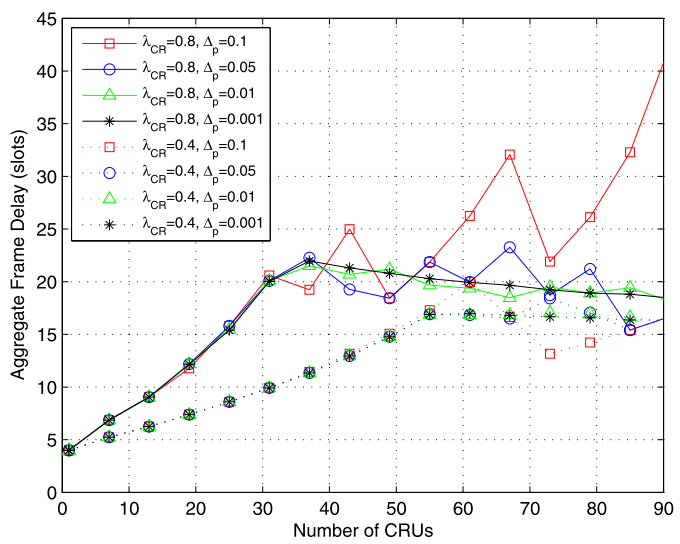

(b) Aggregate frame delay of PUs versus number of CRUs.

Fig. 5. Determination of quantization level $\Delta_{p}$ with $p_{d}=0.93$ and number of channels $M=4$ (each channel with PU's arrival rate $\lambda_{i}=0.1,0.1,0.3,0.3$ for $i=1,2,3,4)$.

\subsection{Performance Validation and Comparison under Paired CR Networks}

\subsubsection{Characterization of Channel-Hopping Probability for a Single Channel}

In this section, the channel-hopping probability will be characterized for the single channel case under paired CR networks. Figs. $6 \mathrm{a}$ and $6 \mathrm{~b}$ illustrate the throughput performance of CRPs and the average frame delay of PU under different values of hopping probability $p_{i}$, respectively. There are total of $N_{p}=14$ CRPs with sensing thresholds $p_{d}=0.93$ and 0.95 under PU's arrival rate $\lambda_{i}=0.05,0.2$, and 0.4 . Noted that the detection probability $p_{d}$ is in general prescribed by primary network in order to regulate the delay constraint for satisfying PU's QoS requirements [22], [26]. However, $P_{d}$ can still be determined by CRUs themselves based on the control of channelhopping sequences. In other words, the QoS requirement in primary network can be achieved by adopting admission control for CRUs. As shown in Fig. 6a, the throughput performance will first increase with $p_{i}$ due to the augmented number of CRPs hopping to the channel which effectively increase the channel utilization. However, the throughput of CRPs decreases with larger $p_{i}$ values owing to insufficient channel availability. Comparing the two values of $p_{d}$, the case with $p_{d}=0.95$ will result in enhanced throughput under larger value of $p_{i}$ since there exists additional CRPs to utilize the channels. On the other hand, with smaller value of $p_{i}$, the detection probability $p_{d}=0.93$ will incur smaller $p_{f a}$ which can allow CRPs to quickly discover the idle slots and consequently increase the channel utilization.

Fig. $6 \mathrm{~b}$ shows that the average frame delay is an increasing function of $p_{i}$ which can become significantly large with increased value of $p_{i}$, i.e., the primary queue can go unstable especially for large PU's arrival probability $\lambda_{i}$ and small sensing threshold $p_{d}$. It can be explained that larger $p_{i}$ corresponds to more CRPs hopping into the $i$ th channel based on $H_{N_{p}, n, i}$ in (3). The transmitted frames from those CRPs will produce more collisions with the PU's frame which makes the increased time in retransmission and therein larger average frame delay in primary network. Moreover, with smaller detection probability $p_{d}$, more collisions from CRPs to PU will be observed. With larger

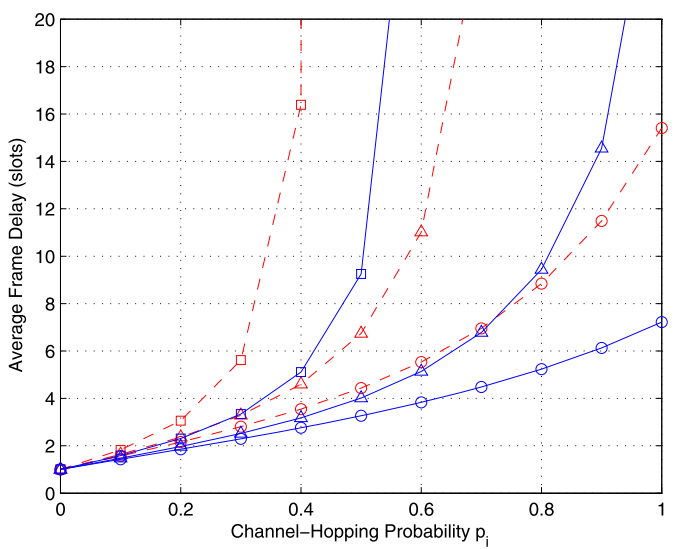

(b) Average frame delay versus channel-hopping probability.

(a) Throughput versus channel-hopping probability.

Fig. 6. Performance of CRPs for a single channel with $p_{d}=0.93$ (dashed line) and $p_{d}=0.95$ (solid line) under PU's arrival rate $\lambda_{i}=0.05,0.2$, and 0.4 denoted by $\circ, \triangle$, and $\square$ curves, respectively. 


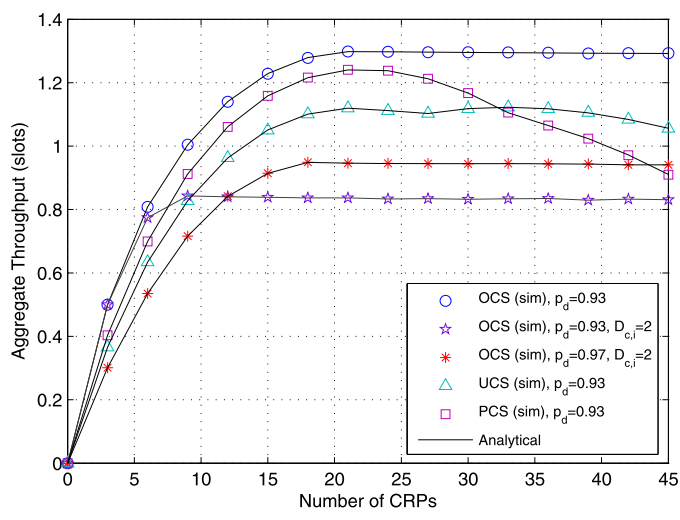

(a) Aggregate throughput versus number of CRPs.

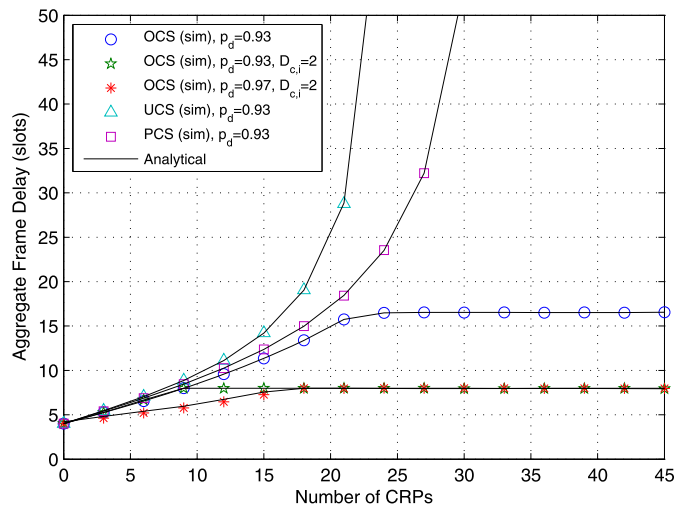

(b) Aggregate frame delay versus number of CRPs.

Fig. 7. Performance validation and comparison under $p_{d}=0.93$ and number of channels $M=4$ with PU's arrival rate at each channel as $\lambda_{i}=0.05,0.05,0.4,0.4$ for $i=1,2,3,4$.

value of $\lambda_{i}$, it is intuitive that long waiting time in the queue will further increase the average frame delay of PU.

\subsubsection{Performance Validation and Comparison}

Two conventional channel-hopping sequences are simulated for comparison purpose as follows: 1) uniform channel-hopping sequence (UCS) with channel-hopping probability $p_{i}=1 / M$ for $i=1,2, \ldots, M$; and 2) proportional channel-hopping sequence (PCS) with channel-hopping probability proportioning to the complement of $\lambda_{i} / \mu$, where $\mu$ represents the service rate. The PCS scheme is designed according to the situation that smaller frame arrival probability is assumed to result in larger channel availability, which can be written as

$$
p_{i}=\frac{1-\frac{\lambda_{i}}{\mu}}{\sum_{i=1}^{M}\left(1-\frac{\lambda_{i}}{\mu}\right)}, \quad i=1,2, \ldots, M .
$$

Noted that the service rate is selected as $\mu=1$ frame/slot which is the stationary idle probability under no collision between the $\mathrm{CR}$ and primary network. For validation purpose, it can be seen from both Figs. $7 \mathrm{a}$ and $7 \mathrm{~b}$ that the simulation results can match with the analytical results for all the three approaches. Fig. 7a shows that the proposed OCS can provide higher aggregate throughput compared to the other channel-hopping sequences since it can exactly exploit the potential throughput in multiple channels. It is noticed that the aggregate throughput in OCS will saturate after exceeding a certain number of CRPs where the number of CRPs is large enough to utilize each channel with optimal throughput. With excessive CRPs in the network, the OCS approach will assign those additional CRPs into the virtual channel in order to reduce packet collision with PU.

On the other hand, as shown in Fig. $7 \mathrm{~b}$, the aggregate frame delay of primary network can be limited by adopting the proposed OCS approach; while that for the other two conventional schemes become significantly large. Even though the delay constraints is not taken into consideration, the OCS approach can still confine the aggregate frame delay to be less than 16 slots under different numbers of CRPs. In order to provide tighter QoS requirement for PUs, the cases with delay constraint $D_{c, i}=2$ for $i=1,2,3,4$ are also shown in both Figs. $7 \mathrm{a}$ and $7 \mathrm{~b}$. With $D_{c, i}=2$, the aggregate frame delay of PUs can be effectively constrained along with lowered aggregated throughput of CRPs. Moreover, considering different sensing thresholds $p_{d}$ with the same $D_{c, i}=2$, large $p_{d}$ will provide enhanced aggregate throughput than small $p_{d}$ case if there exists larger amount of CRPs to exploit the channel availability. On the other hand, under smaller number of CRPs, the OCS scheme with $p_{d}=0.93$ case results in higher aggregate throughput compared to that with $p_{d}=0.97$. The reason is that the case with $p_{d}=0.93$ will incur smaller $p_{f a}$ which can provide CRP to quickly discover the idle slots and consequently increases channel utilization.

Furthermore, as shown in Fig. 7a, the aggregate throughput of PCS algorithm is higher than that of UCS scheme under lower number of CRPs. The reason is that more CRPs are allocated by the PCS method into channels with lower arrival probabilities compared to the UCS scheme. On the other hand, with larger number of CRPs, the aggregate throughput will degrade quickly by adopting the PCS scheme owing to additional packet collisions between CRPs. Moreover, as in Fig. 7b, the UCS scheme will result in the largest aggregate frame delay compared to the other algorithms. The primary network will quickly go unstable due to excessive allocation of CRPs into the channels with larger arrival probabilities. As a result, the merits of adopting OCS approach under paired CR networks can be observed.

\subsection{Performance Validation and Comparison under Generalized CR Networks}

\subsubsection{Performance Validation of Proposed OCS Approach}

Figs. $8 \mathrm{a}$ and $8 \mathrm{~b}$ show performance validation between the simulation and analytical results by adopting proposed OCS scheme under generalized CR networks. Considering different arrival rates $\lambda_{C R}$ of CRUs, the sensing threshold is selected as $p_{d}=0.93$ and the number of channels is $M=4$ with PU's arrival rate at each channel as $\lambda_{i}=0.1,0.1,0.3,0.3$ for $i=1$ to 4 . As in Fig. 8a, the aggregate throughput increases with the number of CRUs and decreases at higher number of CRUs due to insufficient channel availability. 


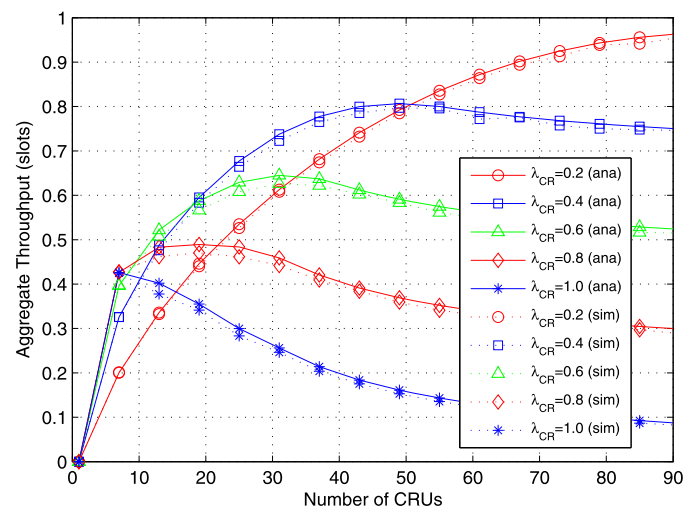

(a) Aggregate throughput versus number of CRUs.

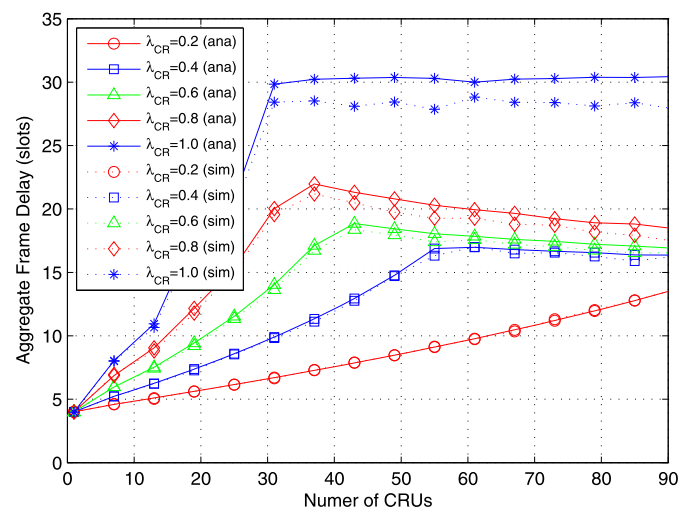

(b) Aggregate frame delay versus number of CRPs.

Fig. 8. Performance validation of OCS scheme under $p_{d}=0.93$ and number of channels $M=4$ with PU's arrival rate at each channel as $\lambda_{i}=0.1,0.1,0.3,0.3$ for $i=1,2,3,4$.

Lowered aggregate throughput is observed at high CR traffic, e.g., at $\lambda_{C R}=1$, due to the decreased rendezvous probability $p_{v, i}$. The CR transmitters do not have much chance to meet with their corresponding $C R$ receivers since those receivers may also conduct data transmission in other channels.

Moreover, as shown in Fig. 8b, the aggregate frame delay in primary network will increase with the augmentation of CR traffic. It can be observed that the peak value of aggregate frame delay will be reached at a larger number of CRUs than that of the aggregate throughput. For instance, under $\lambda_{C R}=0.8$, the maximum delay occurs at the number of CRUs $=38$; while the maximum throughput happens at the number of CRUs $=15$. The concept of proposed OCS scheme is to consider the tradeoff between the rendezvous probability and the channel availability. In other words, the major target is to compromise the rendezvous probability for resolving the logical partition problem, i.e., by increasing the number of CRUs in a channel, and the channel availability by decreasing the number of CRUs in a channel. Between the number of CRUs equal to 15 and 38 , the OCS approach will continue to increase the number of CRUs in a channel in order to augment the rendezvous probability. It indicates that the effect of rendezvous probability will dominate the channel availability, which results in the increased PU's aggregate frame delay. On the other hand, at higher number of CRUs, e.g., the number of CRUs $>38$, the channel availability becomes more important than the rendezvous probability which makes the proposed OCS scheme decrease the number CRUs in a channel. The aggregate frame delay can consequently be decremented as shown in Fig. 8b.

Furthermore, with higher arrival rate $\lambda_{C R}=1$ from CRUs, the PU's aggregate frame delay will not be decreased after reaching the maximum value compared to the cases with lower $\lambda_{C R}$. The reason is mainly due to the excessive CR traffic in the channels. Even though the OCS scheme decreases the number of CRUs in a channel, it is still difficult to increase channel availability which makes PU's aggregate frame delay remain at the same level. It can also be observed from both figures that the analytical results can match with the simulation results in most cases, where the slight discrepancies are resulted from the approximation of binomial distribution adopted in (4), (5), and (28). The larger variation from the approximation occurs under the situations with higher number of CRUs and low rendezvous probability, e.g., as shown in Fig. $8 \mathrm{~b}$ under $\lambda_{C R}=1$ with the number of CRUs $>30$.

\subsubsection{Performance Comparison}

Figs. 9 and 10 show the performance comparison between the proposed OCS approach and the conventional UCS and PCS schemes under the generalized CR networks, where three cases of CRUs' arrival rates $\lambda_{C R}=0.1,0.4$, and 0.7 are illustrated. Compared to the conventional schemes, it is observed from both figures that the proposed OCS algorithm can provide higher aggregate throughput of CRUs with lowered PU's aggregate frame delay. As shown in Fig. 9 under light CR traffic with $\lambda_{C R}=0.1$, the proposed OCS scheme increases the rendezvous probability that effectively allocates the CR transmitters into more feasible channels in order to provide higher channel utilization. With larger CRUs' arrival rate, i.e., $\lambda_{C R}=0.4$, higher
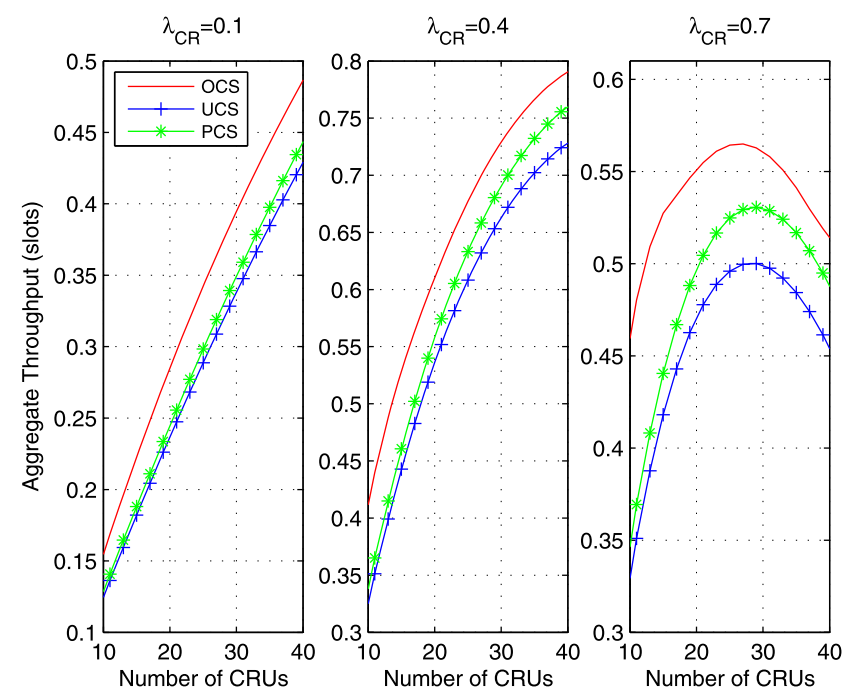

Fig. 9. Performance comparison: aggregate throughput versus number of CRUs with $p_{d}=0.93$ and number of channels $M=4$ with PU's arrival rate at each channel as $\lambda_{i}=0.1,0.1,0.3,0.3$ for $i=1,2,3,4$. 

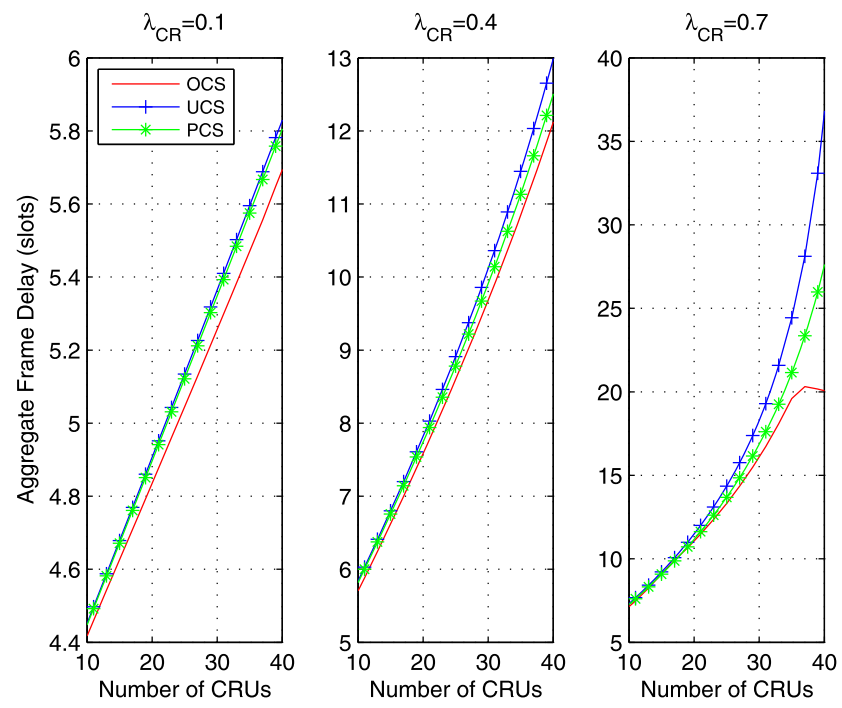

Fig. 10. Performance comparison: aggregate frame delay versus number of CRUs with $p_{d}=0.93$ and number of channels $M=4$ with PU's arrival rate at each channel as $\lambda_{i}=0.1,0.1,0.3,0.3$ for $i=1,2,3,4$.

aggregate throughput is obtained by adopting these three approaches since higher channel utilization can be achieved with feasible amount of CR traffic in the network, e.g., the aggregate throughput of OCS approach can reach around 0.79 slots under the number of CRUs equal to 40 . Furthermore, as $\lambda_{C R}$ reaches 0.7 , the proposed OCS scheme can still outperform the other two methods even though the aggregate throughput is reduced in all three schemes owing to the decrement of rendezvous probability $p_{v, i}$. On the other hand, as illustrated in Fig. 10, the proposed OCS approach can provide lowered PU's aggregate frame delay compared to the other two schemes. Especially under high CRUs' arrival rate with $\lambda_{C R}=0.7$, the OCS scheme can limit the PU's maximum frame delay by reaching to a saturated value of 20 time slots.

\subsubsection{Enhancement with WSC and WCSC Mechanisms}

Figs. 11 and 12 show the comparison between the proposed OCS, OCS-WSC, and OCS-WCSC approaches in generalized CR network under different detection probabilities $p_{d}$ and contention window sizes $W$, i.e., $p_{d}=0.93$ and $W=64$ for Fig. 11 and $p_{d}=0.96$ and $W=16$ for Fig. 12. As shown in the left plots of both Figs. 11 and 12, the proposed OCSWSC and OCS-WCSC mechanisms can always outperform the original OCS approach in aggregate throughput especially in heavy $C R$ traffic owing to the increased rendezvous probability between CRUs. The benefit of OCSWSC and OCS-WCSC schemes can also be revealed in the right plots of both figures that they will not incur additional PU's aggregate frame delay with the enhancement of aggregate throughput.

With the counter-reset mechanism, it is intuitive that the OCS-WCSC scheme can provide more opportunities in the channel negotiation process compared to the OCS-WSC approach as illustrated in Fig. 11. However, with higher $p_{d}$ and smaller contention window $W$ as in Fig. 12, higher collision probability between CRUs will be induced by adopting the OCS-WCSC scheme since more CRUs will be allocated to the channels for increasing channel utilization.
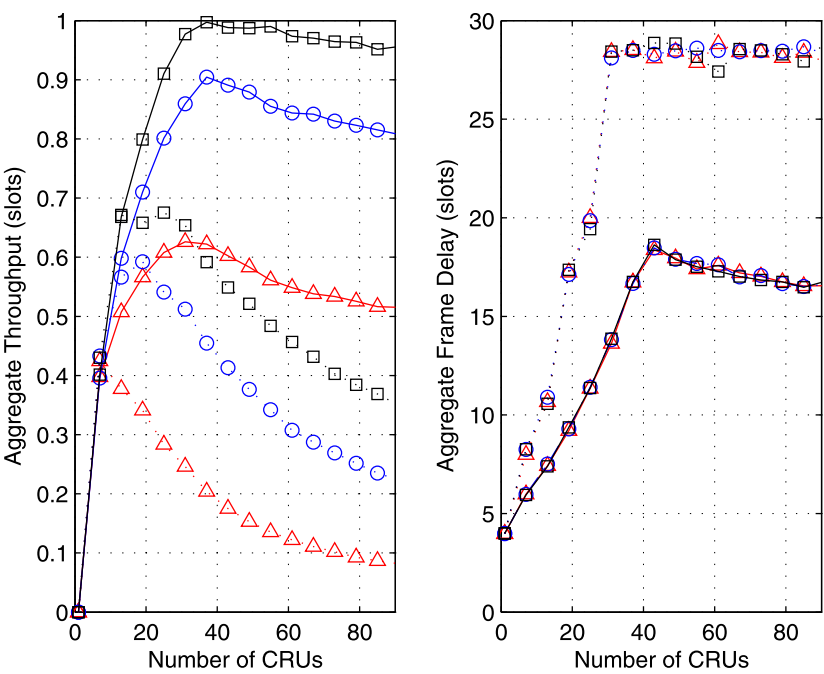

Fig. 11. Performance comparison between proposed algorithms: (left plot) aggregate throughput of CRUs versus number of CRUs, (right plot) aggregate frame delay of PUs versus number of CRUs. Parameters for simulations: $p_{d}=0.93, W=64$, and number of channels $M=4$ with PU's arrival rate $\lambda_{i}=0.1,0.1,0.3,0.3$ for $i=1,2,3,4$. The OCS, OCSWSC, and OCS-WCSC schemes are denoted by $\triangle$, o, and $\square$ curves, respectively, with $\lambda_{C R}=1$ (dashed line) and $\lambda_{C R}=0.6$ (solid line).

Consequently, the aggregate throughput from OCS-WCSC scheme will be comparably smaller than that from the OCSWSC mechanism. It can be observed that both the OCSWSC and OCS-WCSC schemes will outperform the original OCS approach under generalized CR network. The feasible circumstance for adopting either the OCS-WSC or OCSWCSC approach will depend on the system parameters, especially the detection probability $p_{d}$ and the contention window size $W$. As a result, the proposed OCS-WSC and OCS-WCSC schemes can be implemented by constructing offline look-up table based on the key system parameters,
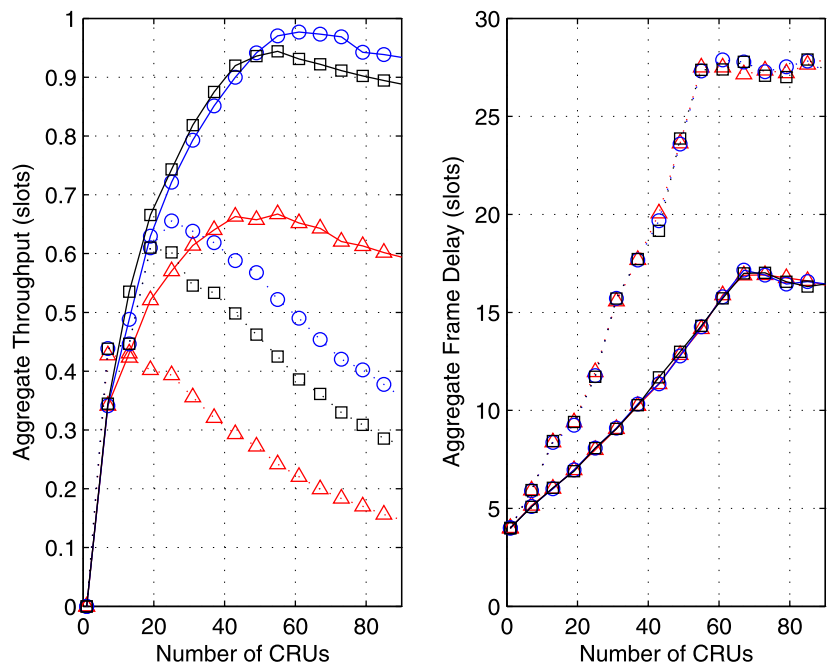

Fig. 12. Performance comparison between proposed algorithms: (left plot) aggregate throughput of CRUs versus number of CRUs, (right plot) aggregate frame delay of PUs versus number of CRUs. Parameters for simulations: $p_{d}=0.96, W=16$, and number of channels $M=4$ with PU's arrival rate $\lambda_{i}=0.1,0.1,0.3,0.3$ for $i=1,2,3,4$. The OCS, OCSWSC, and OCS-WCSC schemes are denoted by $\triangle$, o, and $\square$ curves, respectively, with $\lambda_{C R}=1$ (dashed line) and $\lambda_{C R}=0.6$ (solid line). 
including the number of CRUs $N_{u}$, the CR traffic $\lambda_{C R}$, the probability of detection $p_{d}$, and the contention window size $W$. Afterwards, the table look-up process can be executed for realtime implementation. The benefits of proposed approaches can therefore be observed.

\section{ConCLUSION}

In this paper, a multichannel primary network with the existence of CR users is considered under imperfect spectrum sensing and synchronization. Analytical models are developed for both the probability of channel availability of CR users and the average frame delay of PUs under paired and generalized CR networks. Based on the analysis, an approach for obtaining the optimal channelhopping sequence is designed based on the dynamic programming technique. The proposed OCS approach can both achieve maximum aggregate throughput of CR users and ensure feasible average frame delay of PUs under their QoS requirements. Moreover, the wake-up successive contention and the wake-up counter-reset successive contention algorithms are proposed in order to alleviate the logic partition problem occurs in generalized CR network. By exploring a blind spot in the imperfect sensing and amending the conventional contention mechanisms, the proposed schemes can enhance the original OCS approach with increased number of channel negotiations between CR users. Both the analytical and simulation results show that the proposed OCS, OCS-WSC, and OCS-WCSC approaches can effectively enhance the aggregate throughput of CR users with satisfactory aggregate frame delay of PUs.

\section{ACKNOWLEDGMENTS}

This work was in part funded by the Aiming for the Top University and Elite Research Center Development Plan, NSC 99-2628-E-009-005, NSC 98-2221-E-009-065, the MediaTek research center at National Chiao Tung University, and the Telecommunication Laboratories at Chunghwa Telecom Co. Ltd, Taiwan.

\section{REFERENCES}

[1] Fed. Comm. Commission, "Spectrum Policy Task Force Report, FCC 02-155," Nov. 2002.

[2] S. Haykin, "Cognitive Radio: Brain-Empowered Wireless Communications," IEEE J. Selected Areas in Comm., vol. 23, no. 2, pp. 201-220, Feb. 2005.

[3] C.R. Stevenson, G. Chouinard, Z. Lei, W. Hu, S.J. Shellhammer, and W. Caldwell, "IEEE 802.22: The First Cognitive Radio Wireless Regional Area Network Standard," IEEE Comm. Magazine, vol. 47, no. 1, pp. 130-138, Jan. 2009.

[4] IEEE 802.22 Wireless RAN, Std., Functional Requirements for the 802.22 WRAN Standard, IEEE 802.22- 05/0007r46, IEEE, Oct. 2005.

[5] I.F. Akyildiz, W.Y. Lee, M.C. Vuran, and S. Mohanty, "Next Generation/Dynamic Spectrum Access/Cognitive Radio Wireless Networks: A Survey," Computer Networks, vol. 50, pp. 2127-2159, Sept. 2006.

[6] S. Srinivasa and S.A. Jafar, "How Much Spectrum Sharing Is Optimal in Cognitive Radio Networks?" IEEE Trans. Wireless Comm., vol. 7, no. 10, pp. 4010-4018, Oct. 2008.

[7] A.C.-C. Hsu, D.S.L. Wei, and C.-C.J. Kuo, "A Cognitive MAC Protocol Using Statistical Channel Allocation for Wireless Ad-Hoc Networks," Proc. IEEE Wireless Comm. and Networking Conf. (NWCNC), pp. 105-110, Mar. 2007.
[8] A.L. Yau, P. Komisarczuk, and P.D. Teal, "On Multi-Channel MAC Protocols in Cognitive Radio Networks," Proc. Australasian Telecomm. Networks and Applications Conf. (ATNAC), pp. 300-305, Dec. 2008.

[9] Q. Zhao, L. Tong, A. Swami, and Y. Chen, "Decentralized Cognitive MAC for Opportunistic Spectrum Access in Ad Hoc Networks: A POMDP Framework," IEEE J. Selected Areas in Comm., vol. 25, no. 3, pp. 589-600, Apr. 2007.

[10] J. Jia, Q. Zhang, and X. Shen, "HC-MAC: A HardwareConstrained Cognitive MAC for Efficient Spectrum Management," IEEE J. Selected Areas in Comm., vol. 26, no. 1, pp. 106-117, Jan. 2008.

[11] H. Su and X. Zhang, "Channel-Hopping Based Single Transceiver MAC for Cognitive Radio Networks," Proc. 42nd Ann. Conf. Information Sciences and Systems (CISS), pp. 197-202, Mar. 2008.

[12] L. Le and E. Hossain, "OSA: A MAC Protocol for Opportunistic Spectrum Access in Cognitive Radio Networks," Proc. IEEE Wireless Comm. and Networking Conf. (WCNC), pp. 1426-1430, Apr. 2008.

[13] J. Cai and A.S. Alfa, "Optimal Channel Sensing in Wireless Communication Networks with Cognitive Radio," Proc. IEEE Int'l Conf. Comm. (ICC), pp. 1-5, June 2009.

[14] C. Xin and X. Cao, "A Cognitive Radio Network Architecture without Control Channel," Proc. IEEE GlobeCom, pp. 1-6, Dec. 2009.

[15] P. Bahl, R. Chandra, and J. Dunagan, "SSCH: Slotted Seeded Channel Hopping for Capacity Improvement in IEEE 802.11 Ad-Hoc Wireless Networks," Proc. ACM MobiCom, pp. 216-230, 2004.

[16] C.-M. Lee, J.-S. Lin, Y.-P. Hsu, and K.-T. Feng, “Design and Analysis of Optimal Channel-Hopping Sequence for Cognitive Radio Networks," Proc. IEEE Wireless Comm. and Networking Conf. (WCNC), pp. 1-6, Apr. 2010.

[17] J. Gambini, O. Simeone, Y. Bar-Ness, U. Spagnolini, and T. Yu, "Packet-Wise Vertical Handover for Unlicensed Multi-Standard Spectrum Access with Cognitive Radios," IEEE Trans. Wireless Comm., vol. 7, no. 12, pp. 5172-5176, Dec. 2008.

[18] I. Suliman and J. Lehtomaki, "Optimizing Detection Parameters for Time-Slotted Cognitive Radios," Proc. IEEE Vehicular Technology Conf. (VTC), pp. 1-4, Apr. 2009.

[19] “3GPP TR36.814 v9.0.0 Release 9," technical report, Mar. 2010.

[20] Y.-C. Liang, Y. Zeng, E.C.Y. Peh, and A.T. Hoang, "SensingThroughput Tradeoff for Cognitive Radio Networks," IEEE Trans. Wireless Comm., vol. 7, no. 4, pp. 1326-1337, Apr. 2008.

[21] A. Ghasemi and E.S. Sousa, "Spectrum Sensing in Cognitive Radio Networks: Requirements, Challenges and Design Trade-Offs," IEEE Comm. Mag., vol. 46, no. 4, pp. 32-39, Apr. 2008.

[22] S. Zheng, Y.-C. Liang, P.Y. Kam, and A.T. Hoang, "Cross-Layered Design of Spectrum Sensing and MAC for Opportunistic Spectrum Access," Proc. IEEE Wireless Comm. and Networking Conf. (WCNC), pp. 1-6, Apr. 2009.

[23] A. Gravey and G. Hebuterne, "Simultaneity in Discrete-Time Single Server Queues with Bernoulli Inputs," Performance Evaluation, vol. 14, pp. 123-131, Jan. 1992.

[24] T. Meisling, "Discrete-Time Queuing Theory," Operations Research, vol. 6, pp. 96-105, Jan. 1958

[25] D. Gross and C.M. Harris, Fundamentals of Queuing Theory, fourth ed. Wiley, 2008.

[26] A.T. Hoang, D.T.C. Wong, and Y.-C. Liang, "Design and Analysis for an 802.11-Based Cognitive Radio Network," Proc. IEEE Wireless Comm. and Networking Conf. (WCNC), pp. 1-6, Apr. 2009.

[27] IEEE Std 802.11b-1999 (R2003): Part 11: Wireless LAN Medium Access Control (MAC) and Physical Layer (PHY) specifications: Higher-Speed Physical Layer Extension in the 2.4 GHz Band, IEEE, 2003.

[28] A.V. Adamis, K.N. Maliatsos, and P. Constantinou, "Methods for Reducing Interference Caused to Licensed Systems by OverlayCSMA/CA Cognitive Radios," Proc. Third Int'l Conf. Cognitive Radio Oriented Wireless Networks and Comm. (CROWNCOM), pp. 16, May 2008.

[29] D. Bertsekas, Dynamic Programming and Optimal Control, third ed. Athena Scientific, 2005.

[30] L. Gao and S. Cui, "Power and Rate Control for DelayConstrained Cognitive Radios via Dynamic Programming," IEEE Trans. Vehicular Technology, vol. 58, no. 9, pp. 4819-4827, Nov. 2009.

[31] T.H. Cormen, C.E. Leiserson, R.L. Rivest, and C. Steinyd, Introduction to Algorithms, third ed. The MIT Press, 2009. 


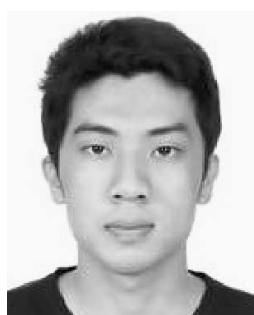

Chi-Mao Lee received the BS degree in 2008 and the MS degree in communication engineering in 2010 from National Chiao Tung University, Hsinchu, Taiwan. His current research interests include wireless local area networks and cognitive radio networks.

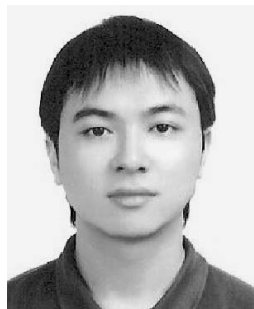

Jia-Shi Lin received the BS degree from National Tsing Hua University, Hsinchu, Taiwan, in 2007 and the MS degree in communication engineering from National Chiao Tung University, Hsinchu, Taiwan in 2009. Since 2009, he has been working toward the $\mathrm{PhD}$ degree in the Department of Electrical Engineering, National Chiao Tung University, Hsinchu, Taiwan. His current research interests include game theory, MAC protocol design, wireless local area networks, and cognitive radio networks. He is a student member of the IEEE.

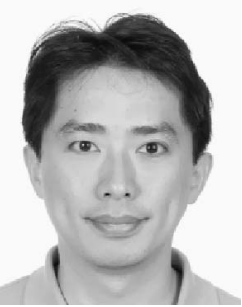

Kai-Ten Feng received the BS degree from National Taiwan University, Taipei, Taiwan, in 1992, the MS degree from the University of Michigan, Ann Arbor, in 1996, and the PhD degree from the University of California, Berkeley, in 2000. Between 2000 and 2003, he was an In-Vehicle Development Manager/Senior Technologist with OnStar Corporation, a subsidiary of General Motors Corporation, where he worked on the design of future telematics platforms and in-vehicle networks. Since August 2011, he has been a full professor with the Department of Electrical Engineering, National Chiao Tung University (NCTU), Hsinchu, Taiwan, where he was an associate professor and assistant professor from August 2007 to July 2011 and from February 2003 to July 2007, respectively. From July 2009 to March 2010, he was a visiting scholar with the Department of Electrical and Computer Engineering, University of California at Davis. He has also been the convener of the NCTU Leadership Development Program since August 2011. Since October 2011, he has served as the director of the Digital Content Production Center at the same university. His current research interests include broadband wireless networks, cooperative and cognitive networks, smart phone and embedded system designs, wireless location technologies, and intelligent transportation systems. He received the Best Paper Award from the Spring 2006 IEEE Vehicular Technology Conference, which ranked his paper first among the 615 accepted papers. He also received the Outstanding Youth Electrical Engineer Award in 2007 from the Chinese Institute of Electrical Engineering and the Distinguished Researcher Award from NCTU in 2008, 2010, and 2011. He has served on the technical program committees of VTC, ICC, and WCNC. He is a member of the IEEE and the IEEE Computer Society.

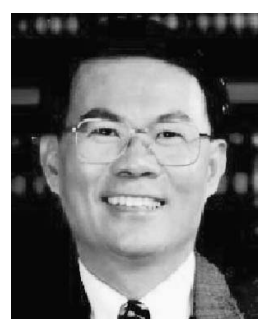

Chung-Ju Chang received the $\mathrm{BE}$ and $\mathrm{ME}$ degrees in electronics engineering from National Chiao Tung University, Hsinchu, Taiwan, in 1972 and 1976, respectively, and the PhD degree in electrical engineering from National Taiwan University, Taiwan, in 1985. From 1976 to 1988 , he was with Telecommunication Laboratories, Directorate General of Telecommunications, Ministry of Communications, Taiwan, as a design engineer, supervisor, project manager, and then division director. $\mathrm{He}$ also acted as a science and technical advisor for the Minister of the Ministry of Communications from 1987 to 1989. In 1988, he joined the Faculty of the Department of Communication Engineering, College of Electrical Engineering and Computer Science, National Chiao Tung University, as an associate professor. He has been a professor since 1993 and a chair professor since 2009. He was the director of the Institute of Communication Engineering from August 1993 to July 1995, chairman of the Department of Communication Engineering from August 1999 to July 2001, and the dean of the Research and Development Office from August 2002 to July 2004. Also, he was an advisor for the Ministry of Education to promote the education of communication science and technologies for colleges and universities in Taiwan during 1995-1999. $\mathrm{He}$ is acting as a committee member of the Telecommunication Deliberate Body, Taiwan. Moreover, he once served as an editor for IEEE Communications Magazine and an associate editor for IEEE Transactions Vehicular Technology. His research interests include performance evaluation, radio resources management for wireless communication networks, and traffic control for broadband networks. He is members of the Chinese Institute of Engineers (CIE) and the Chinese Institute of Electrical Engineers (CIEE). He is a fellow of the IEEE and the IEEE Computer Society.

For more information on this or any other computing topic, please visit our Digital Library at www.computer.org/publications/dlib. 